\title{
A Review of Subthreshold Micropulse Laser for Treatment of Macular Disorders
}

\author{
Paula Scholz (D) Lebriz Altay $\cdot$ Sascha Fauser
}

Received: March 30, 2017 / Published online: May 24, 2017

(C) The Author(s) 2017. This article is an open access publication

\begin{abstract}
Micropulse laser treatment is an alternative to the conventional continuous-wave laser for the treatment of retinal or macular diseases. In contrast to the conventional laser, the therapeutic effect of the subthreshold micropulse laser is not accompanied by thermal retinal damage. This fact is of particular importance when a treatment near the fovea is required. Micropulse treatment is applied in indications such as central serous chorioretinopathy (CSC), diabetic macular edema (DME), or macular edema due to retinal vein occlusion (RVO). This review outlines and discusses the published literature of subthreshold micropulse laser treatment for CSC, DME, and macular edema after RVO.
\end{abstract}

Keywords: Central serous chorioretinopathy; Diabetic macular edema; Micropulse laser;

Enhanced content To view enhanced content for this article go to http://www.medengine.com/Redeem/484 8F0600C509A9C.

P. Scholz $(\bowtie) \cdot$ L. Altay $\cdot$ S. Fauser Department of Ophthalmology, University Hospital of Cologne, Cologne, Germany

e-mail: paula.scholz@uk-koeln.de

S. Fauser

F. Hoffmann-La Roche, Basel, Switzerland
Ophthalmology; Retinal vein occlusion; Subthreshold laser

\section{INTRODUCTION}

Traditional laser photocoagulation has been used to treat different retinal diseases for many years [1-5]. Here, the endpoint is a visible whitening of the retina due to thermal damage of the retinal pigment epithelium (RPE) and the inner retina. However, apart from the favored therapeutic effect, the treatment can lead to undesirable side effects like visual field defects, epiretinal fibrosis, and choroidal neovascularization (CNV) in the area of the laser scar [6-10]. The mechanisms which are responsible for the therapeutic effect are still poorly understood.

Scarring seems not to be necessary to achieve a therapeutic effect. It might be the stimulation of the RPE alone and not the destroying of the photoreceptors that is needed to reach a therapeutic effect of laser photocoagulation [11]. The laser energy stimulates the RPE, which leads to repair of the inner blood retinal barrier [12]. A modification of the gene expression initiated by the wound healing response after laser photocoagulation could be responsible for the beneficial effect of laser photocoagulation. Sublethally injured RPE cells induce an up- and downregulation of various factors [pigment epithelium-derived factor (PEDF), vascular endothelial growth factor (VEGF) inhibitors, 
VEGF inducers, permeability factors, etc.] which restores the pathologic imbalance. RPE cells destroyed by thermal heat are not capable of inducing this biologic activity [13, 14]. Inagaki et al. [15] showed that sublethal photothermal stimulation with a micropulse laser induces heat shock protein expression in RPE cells without cellular damage in a model of human RPE.

In subthreshold micropulse laser (SML), diffusion of heat to surrounding tissues is minimized and thereby scarring is prevented.

The neural retina can be spared by applying the minimum laser irradiance (watts per square meter) needed to raise the temperature of the $\mathrm{RPE}$, but without exceeding the protein denaturation threshold. This leads to the required activation of the RPE cells, but the thermal wave will only reach the neural retina at temperatures beneath the protein denaturation threshold. Since the RPE and the neural retina are close together, the laser pulse has to be in the microsecond range and not in the millisecond range like the traditionally used supra threshold laser. For safety reasons it is not possible to deliver the required energy in one short enough laser pulse. A single laser pulse would require so much energy that there would be a high risk of bubble formation and micro-explosions, accompanied by retinal hemorrhages [16]. Those side effects can be avoided by using a repetitive series of very short pulses with low energy instead of a continuous-wave laser pulse [17-19].

The micropulse operating mode and terminology were described by Dorin [20]. In the traditional continuous-wave mode, a single laser pulse of $0.1-0.5 \mathrm{~s}$ delivers the preset laser energy. In the micropulse mode, a train of repetitive short laser pulses delivers the laser energy within an "envelope" whose width is typically $0.1-0.5 \mathrm{~s}$. The normal length of each pulse is $100-300 \mu \mathrm{s}$. The "envelope" includes "ON" time, which is the duration of each micropulse, and "OFF" time, which is the time between the micropulses. The "OFF" time is important since here the originated heat can cool down. The sum of the "ON" and "OFF" times is the period $T$ and its reciprocal $1 / T$ is the frequency (pulses per second) $f$ in hertz $(\mathrm{Hz})$.
The duty cycle in percent is the ratio between "ON" time and the period $T$.

\section{DIFFERENT LASERS AVAILABLE WITH MICROPULSE MODE}

\section{0-nm Diode Laser}

The commercially available diode lasers emit at a wavelength of $810 \mathrm{~nm}$, which is in the near-infrared range of the spectrum. A feature of the $810-\mathrm{nm}$ wavelength is its deep penetration into the choroid, but it is not clear if this characteristic is relevant in micropulse treatment. For all indications requiring a treatment near the foveal avascular zone, the 810-nm laser has the advantage that the laser energy will relatively spare the inner neurosensory retina and affect mainly the deeper layers [21-24]. The deep penetration is a possible benefit especially for central serous chorioretinopathy (CSC) since the choroid may play a role in the pathogenesis of CSC. A potential disadvantage of the 810-nm laser is a possible sensation of pain during treatment with a diode laser [24, 25], although this is a rare problem in the micropulse mode.

\section{7-nm Yellow Laser}

Another laser type which is available for micropulse treatment is the $577-\mathrm{nm}$ yellow laser. The yellow laser has the advantage that xanthophyll, the pigment which is located in the inner and outer plexiform layers of the macula, absorbs the yellow light only minimally so treatment near the fovea is relatively safe [26].

\section{APPLICATIONS FOR SUBTHRESHOLD MICROPULSE LASERS}

In this article we will review the applications for micropulse laser in macular diseases, namely CSC, diabetic macular edema (DME), and retinal vein occlusion (RVO). We will give an overview of the available literature and outline 
the current evidence for micropulse laser treatment in each field.

The literature search was performed in English language in the PubMed database. We used pairings of the terms "micropulse", "laser", "subthreshold", and "central serous chorioretinopathy", "chorioretinopathy", "central serous retinopathy", or "diabetic macular edema", "macular edema" and "retinal vein occlusion", "branch retinal vein occlusion", "central retinal vein occlusion". Additionally, the references of the resultant articles were checked for publications missing in the primary search. Until February 2017 we found 18 articles [27-44] concerning micropulse laser in CSC; no articles were excluded and all articles are listed in Table 1. As a result of the high number of publications related to DME and micropulse treatment, we only listed the 11 prospective studies [45-55] in Table 2. We found four studies [56-59] investigating micropulse laser for RVO, which are all listed in Table 3.

As a result of different study designs, uneven inclusion and exclusion criteria, different laser types, treatment parameters, and various outcome measures, a direct comparison of the studies is limited. We looked for similarities referring to the outcome measures for making comprehensive conclusions regarding the treatment outcome. In Tables 1, 2, and 3, all studies are listed, but individual studies were excluded from the calculations as a result of missing information or prior treatment. The studies had a high variety regarding the follow-up visits. If available, after calculation of the decrease in central retinal thickness (CRT) in optical coherence tomography (OCT) in all individual studies, a weighted average value was calculated on the basis of the number of patients in each study. The best corrected visual acuity (BCVA) was not consistently presented in the different studies. To compare the BCVA, we converted all visual acuity data to Early Treatment Diabetic Retinopathy Study (ETDRS) letters equivalent using the formula ETDRS letters $=85+50 \times \log$ (Snellen fraction) [60]. If a large enough number of studies provided information about a control group, we additionally analyzed the control group regarding CRT, BCVA, and treatment outcome.
This article was based on previously conducted studies and did not involve any new studies of human or animal subjects performed by any of the authors.

\section{CENTRAL SEROUS CHORIORETINOPATHY (CSC)}

In CSC a serous detachment of the neurosensory retina leads to decreased vision [61]. The acute form of CSC is often self-limiting so that treatment is not always necessary. But some patients develop the chronic form of CSC with impending permanent structural damage and vision loss [62-64]. For patients with extrafoveal leakage, a continuous-wave laser photocoagulation is a treatment option. Studies showed an acceleration of subretinal fluid (SRF) resolution but no change in final visual acuity or recurrence rate after conventional laser. Furthermore, adverse events like CNV, scotomas, enlargement of the laser spot, and reduction of contrast sensitivity can occur [3, 62, 65-67]. Another treatment option is photodynamic therapy (PDT) which is used also in juxtafoveal or subfoveal leakage. But even with reduced treatment settings, complications like RPE atrophy, choroidal hypoperfusion, transient reduction of macular function, and CNV can occur [68-71].

Bandello et al. [72] presented the first pilot study investigating SML treatment for CSC in 2003. They reported a high treatment success with complete resorption of SRF in five out of five eyes within 1 month and no recurrence of SRF during follow-up of 2-6 month after non-visible subthreshold micropulse diode laser $(810 \mathrm{~nm})$ treatment. No evidence of RPE or retinal changes was discernible at fluorescein angiography (FA) or fundus biomicroscopy after laser treatment.

Table 1 shows all identified studies investigating micropulse laser treatment for CSC. In Table 4, the treatment outcome after SML, PDT, and observation for CSC is presented.

\section{Treatment Response}

Most studies defined a treatment response as a reduction in CRT measured in spectral domain 


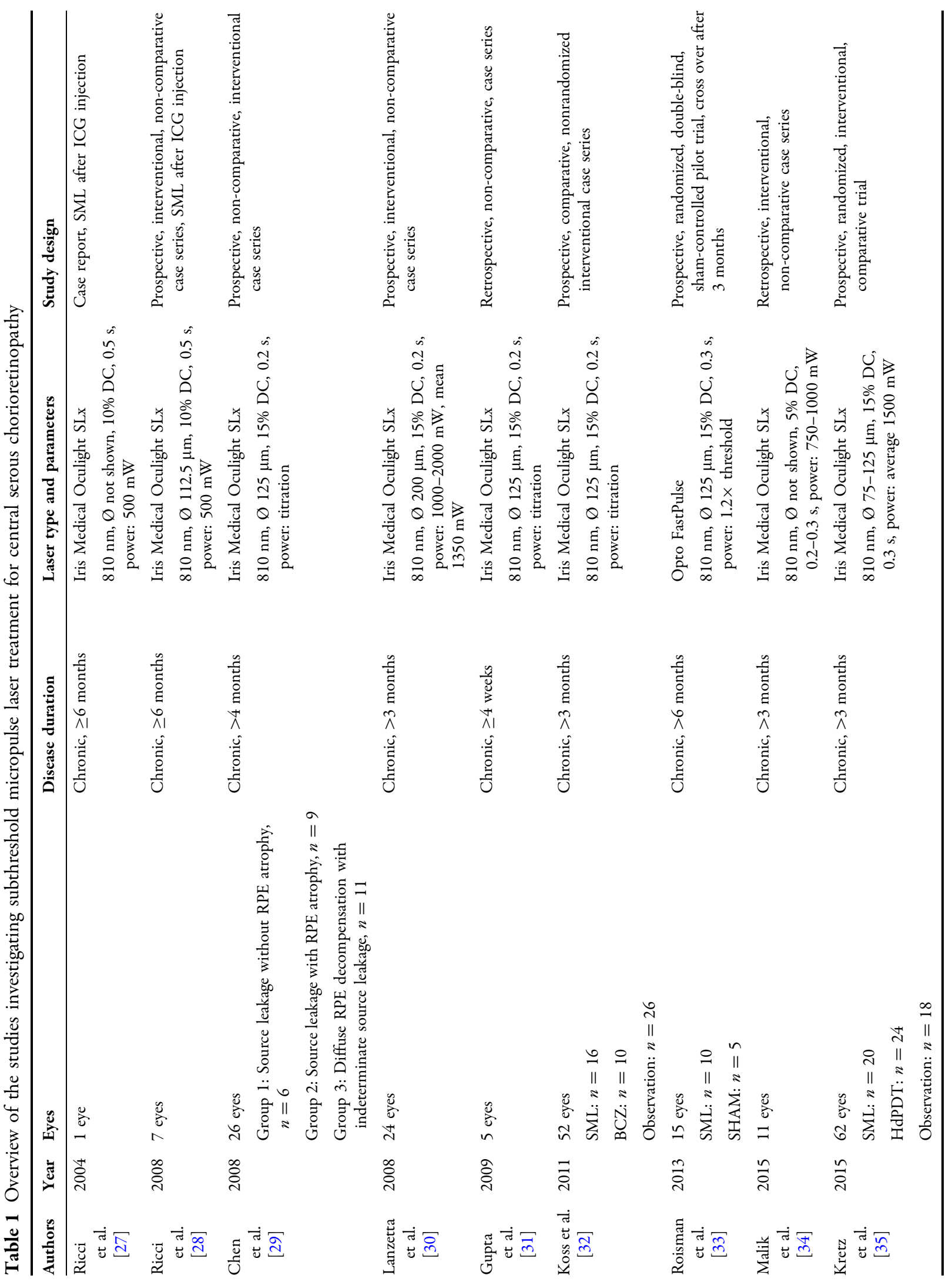




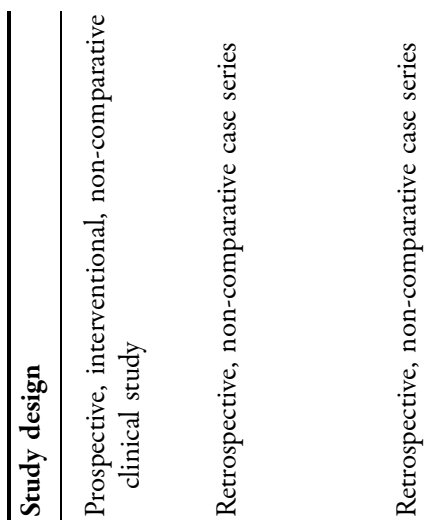

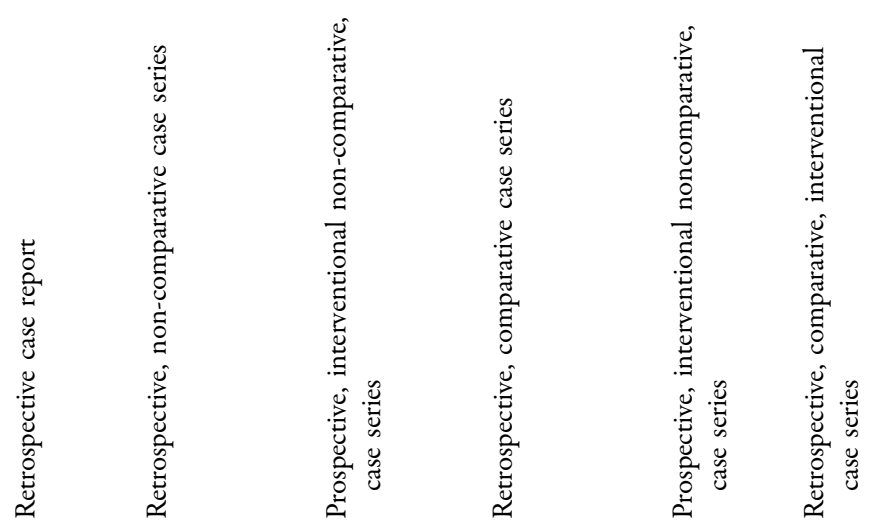

$\dot{3}$
$\hat{0}$
$\hat{u}$

$\begin{array}{ccc}\hat{n} & \hat{n} & \hat{0} \\ \tilde{0} & 0 & 0 \\ 0 & 0 & 0\end{array}$

$\begin{array}{lll}\hat{n} & \hat{0} \\ 0 & 0 & 0 \\ 0 & 0 & 0\end{array}$

نी

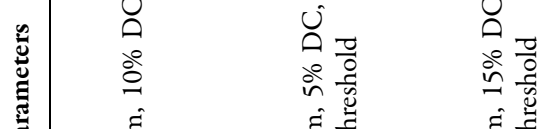

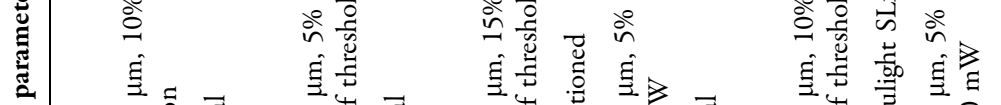

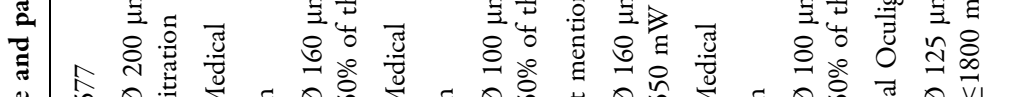

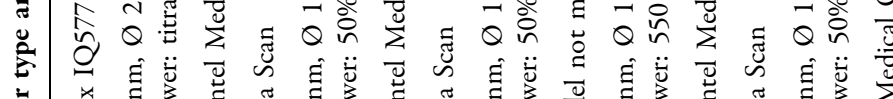

is

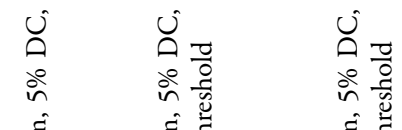

ì

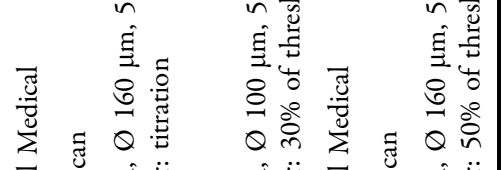

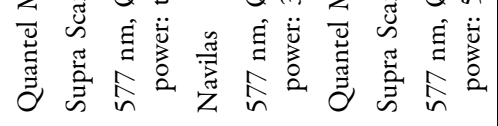

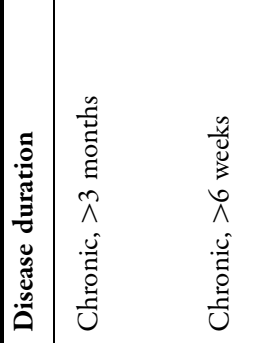
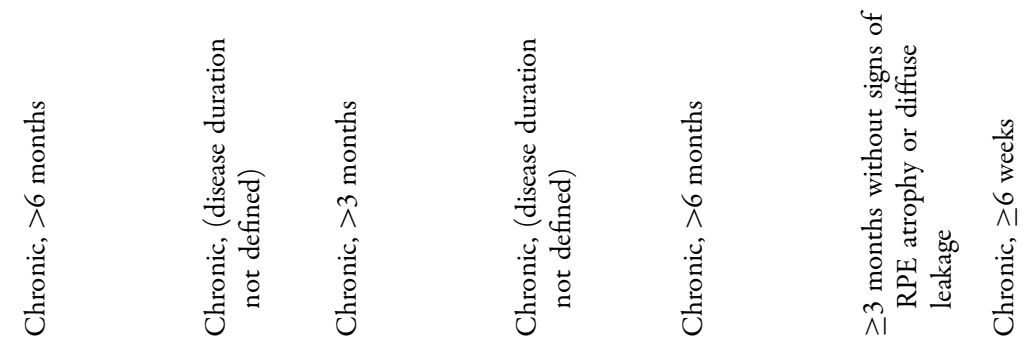

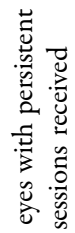

온

完西

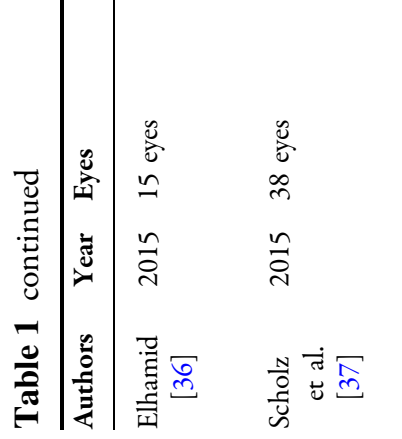

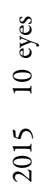
$\stackrel{0}{0}$

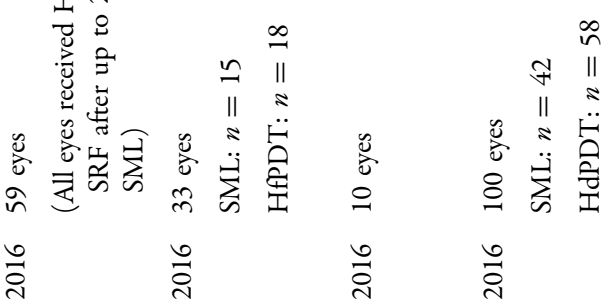

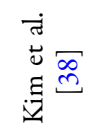
กั่
ำ

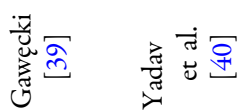
竘苛可
节悹导

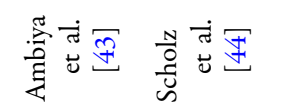




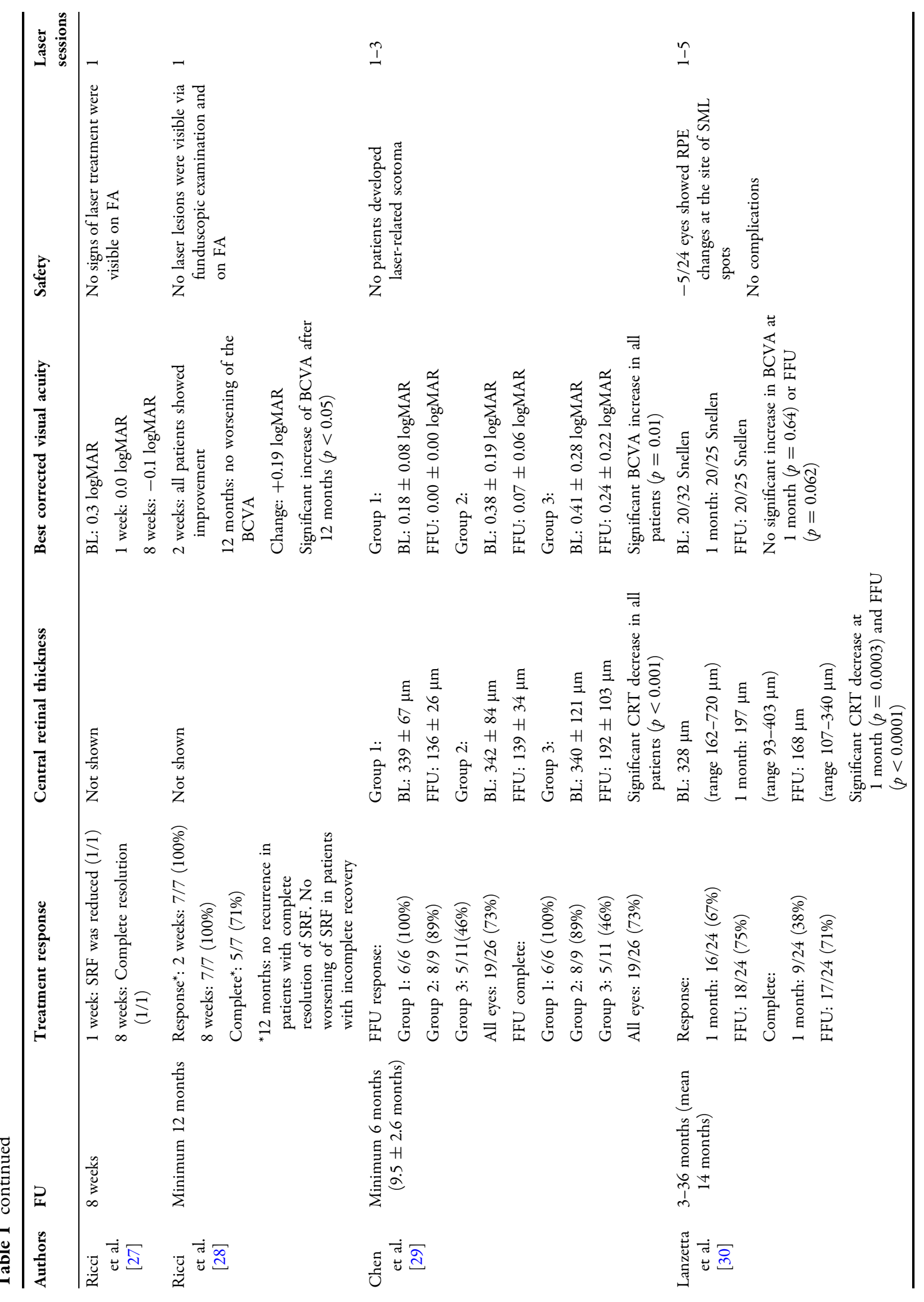




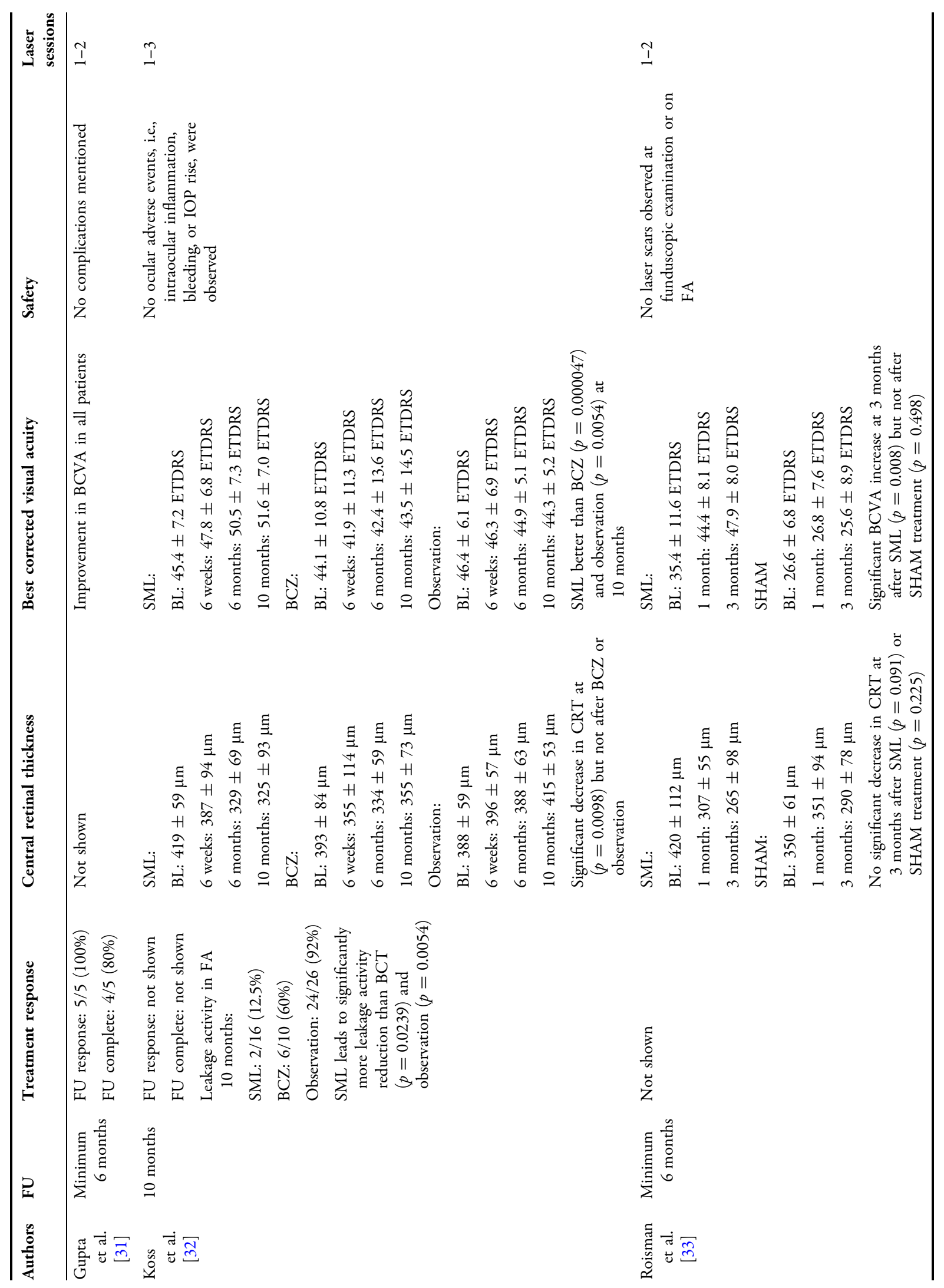




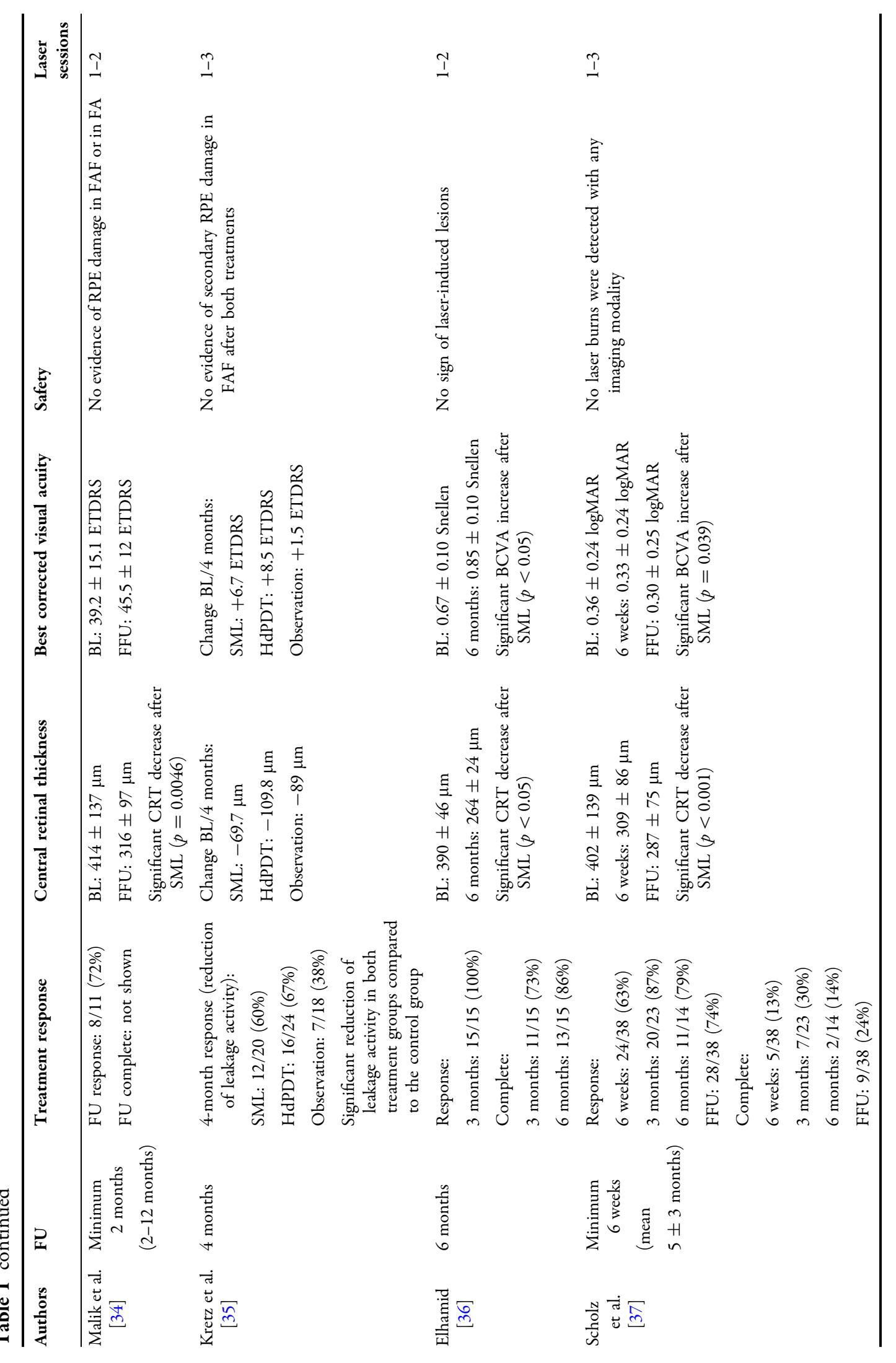




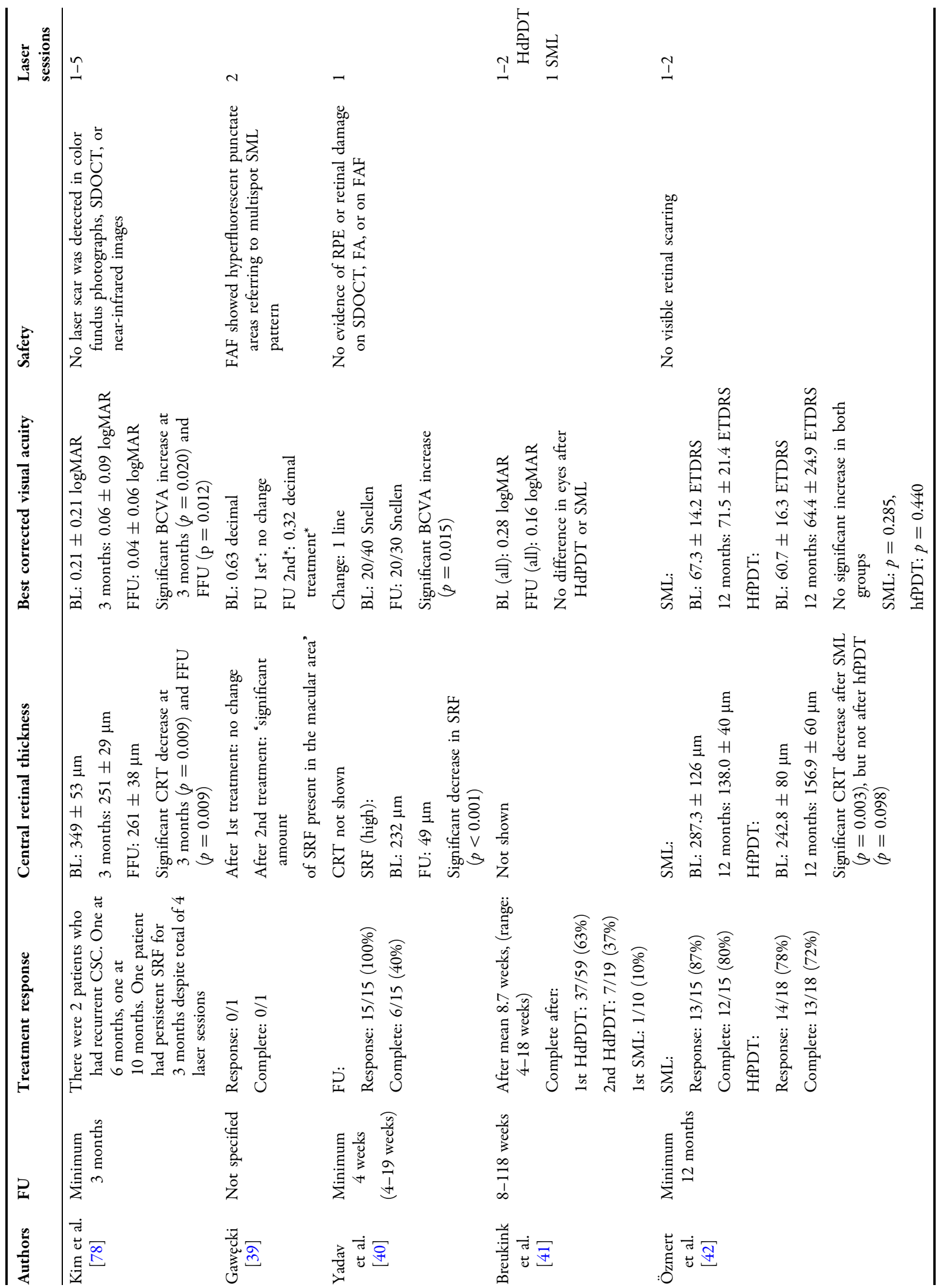




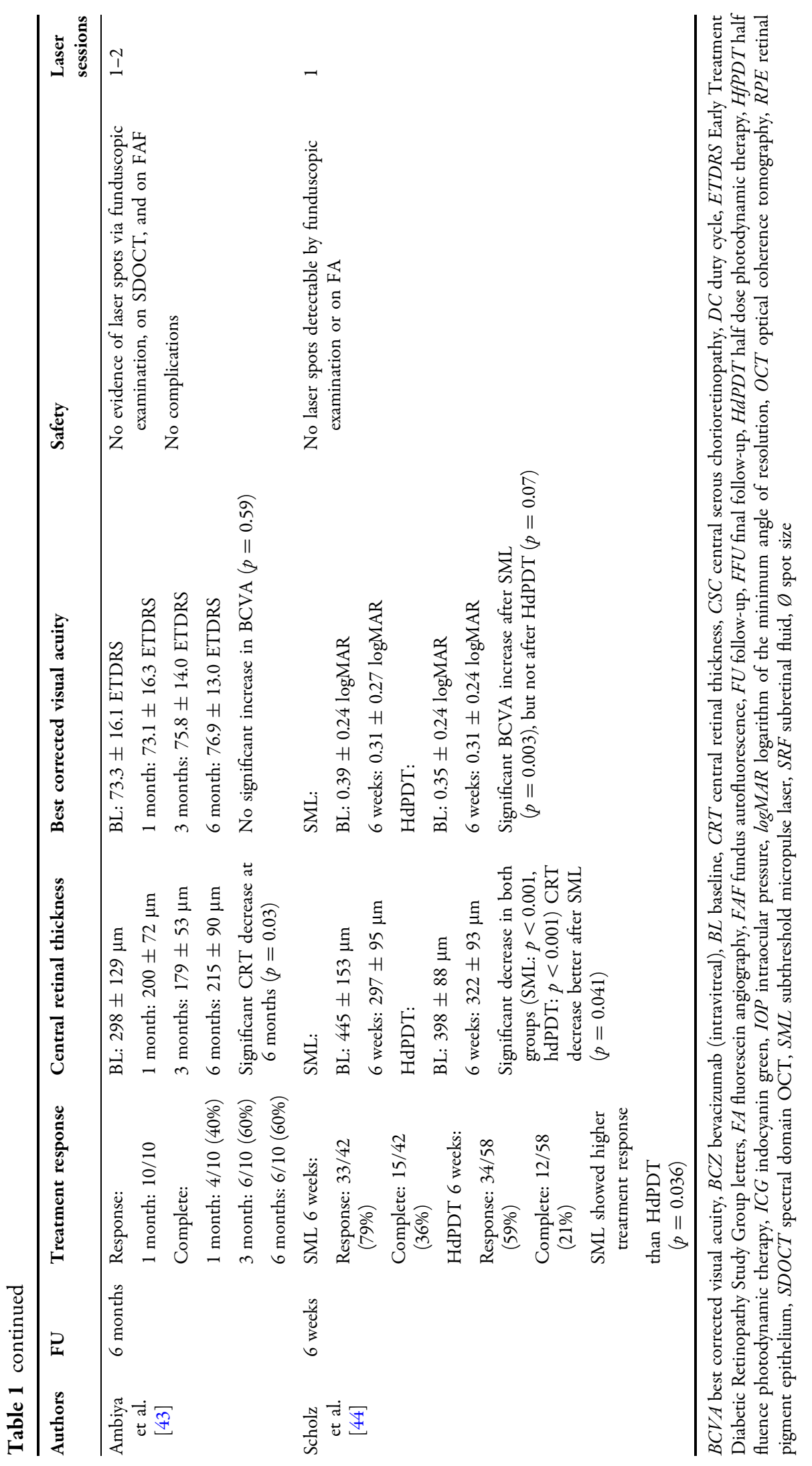




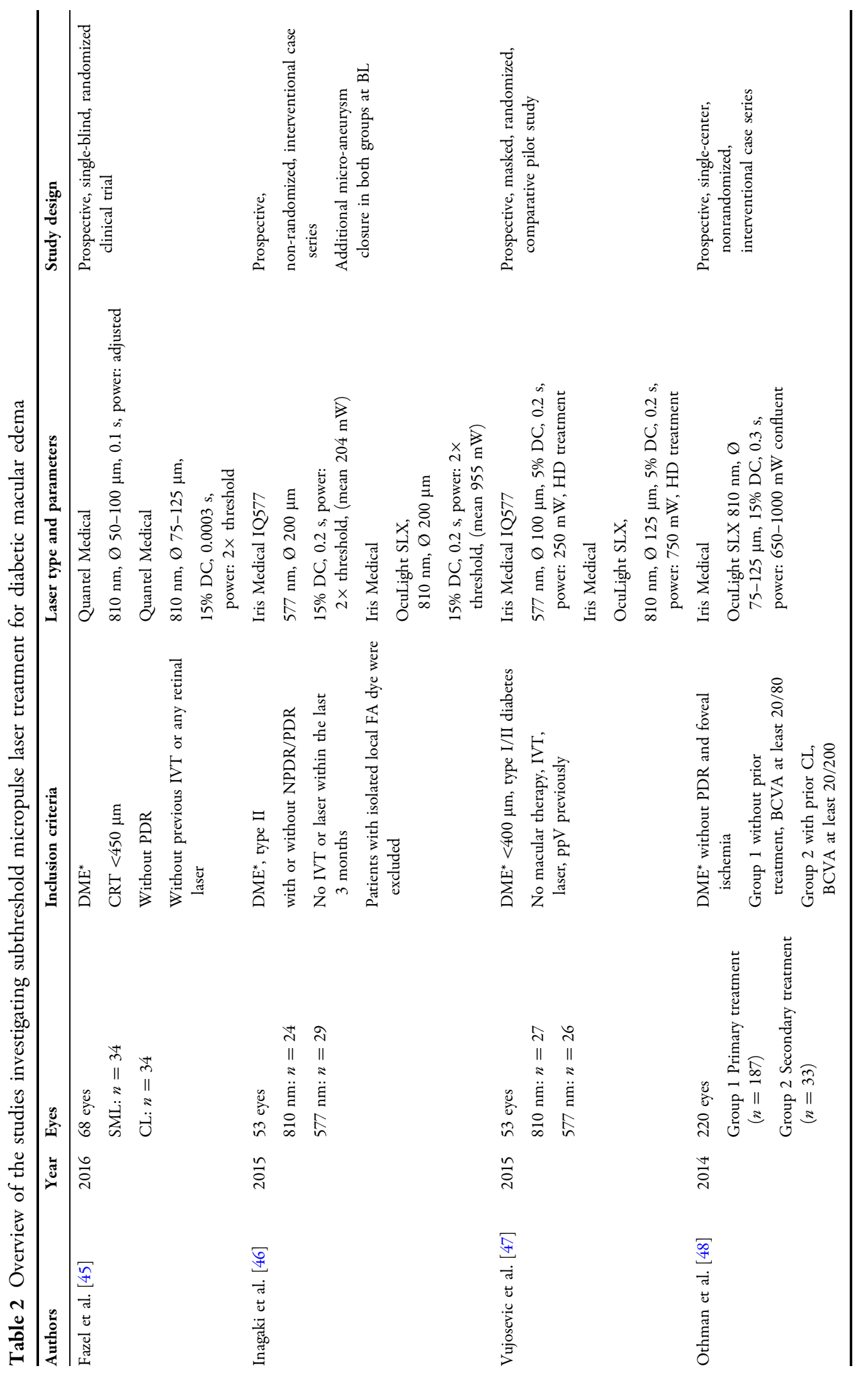




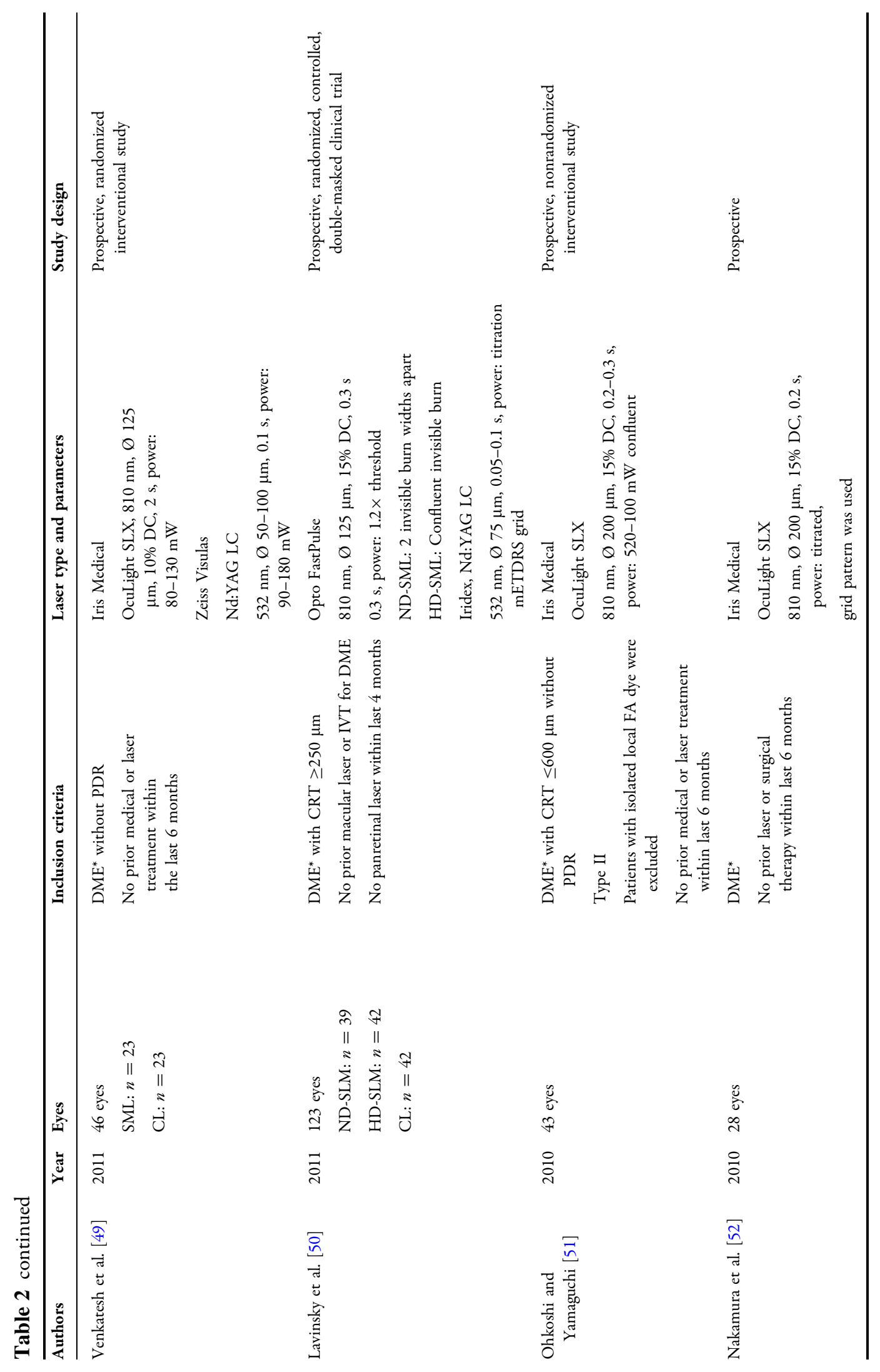




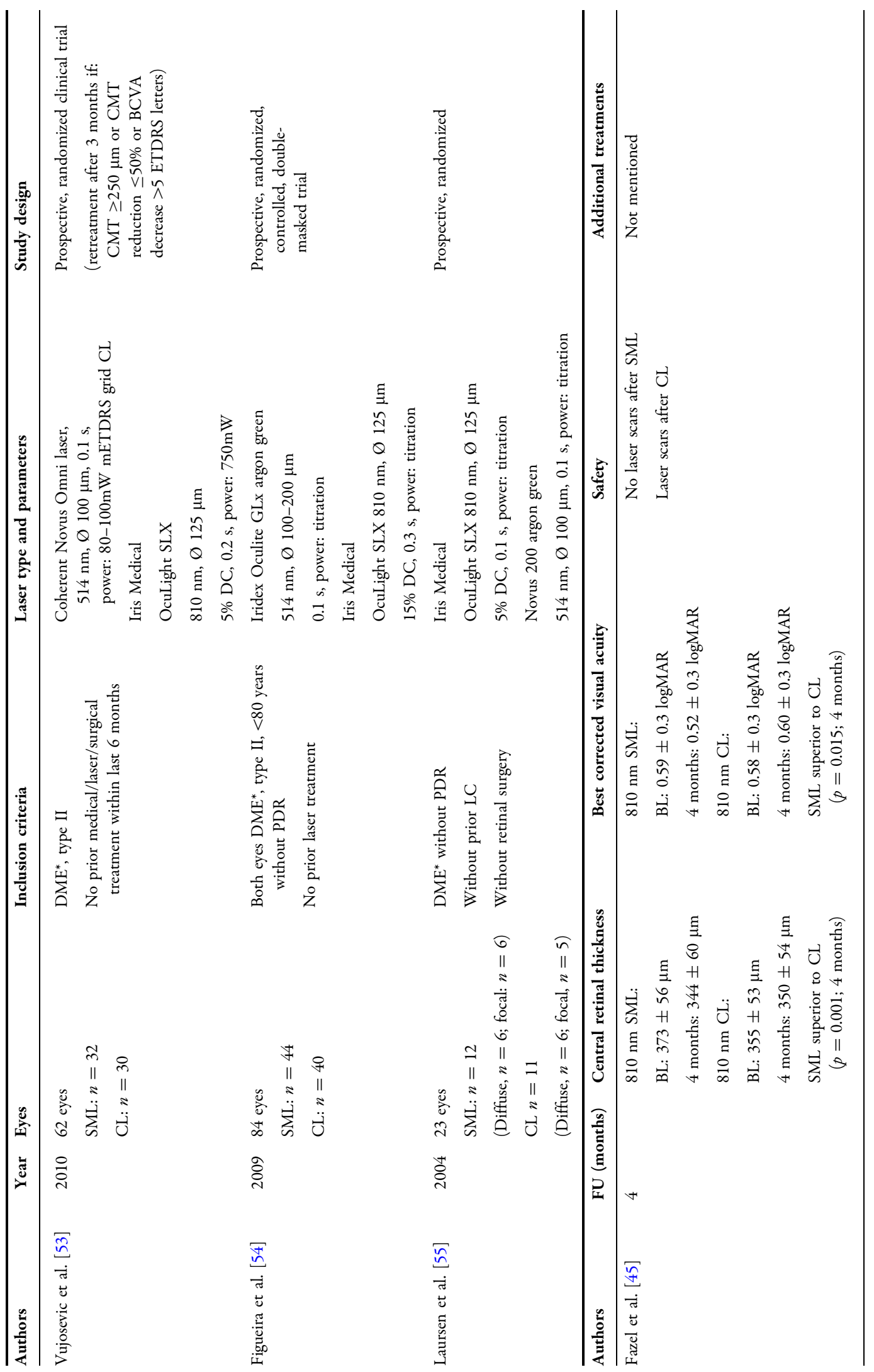




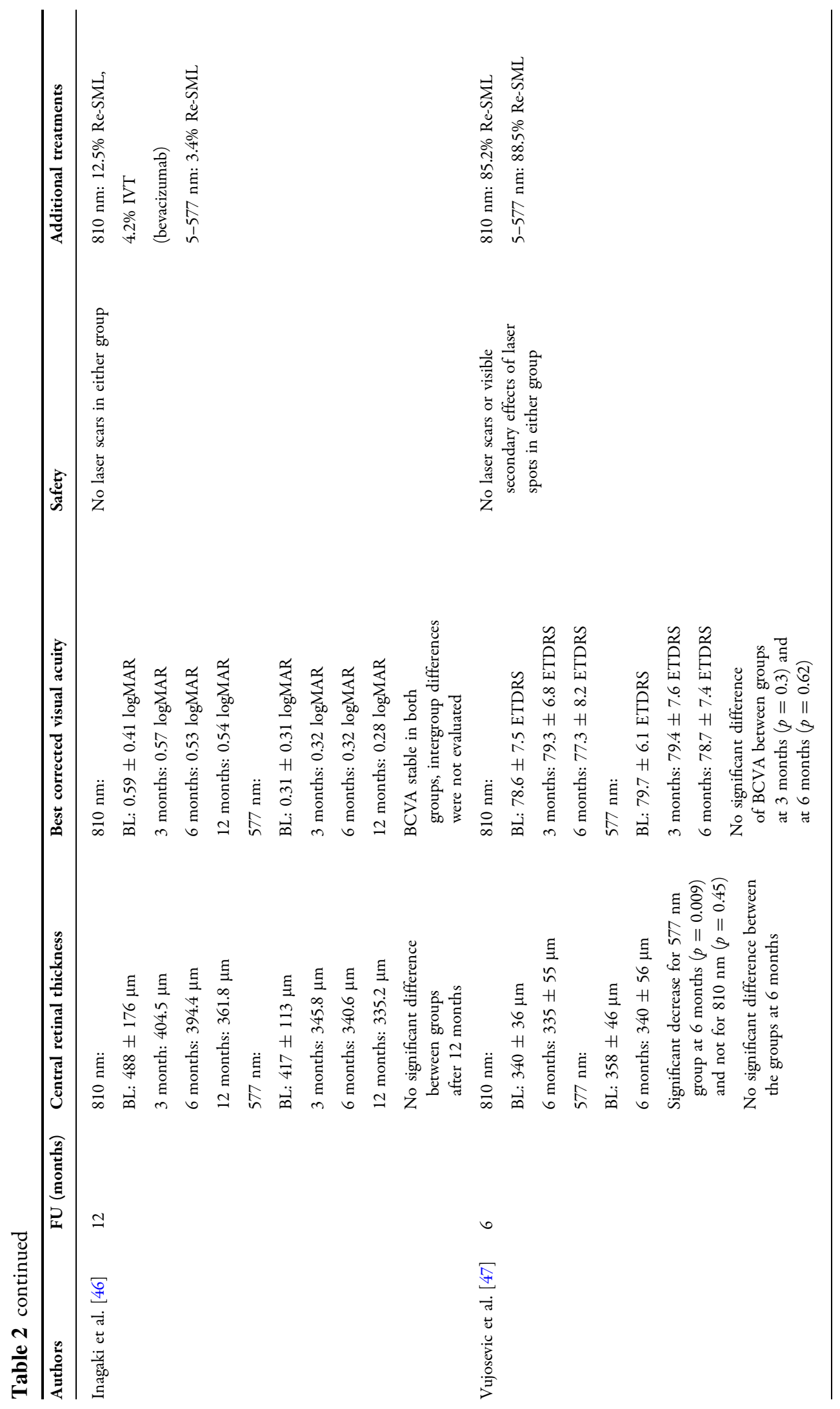




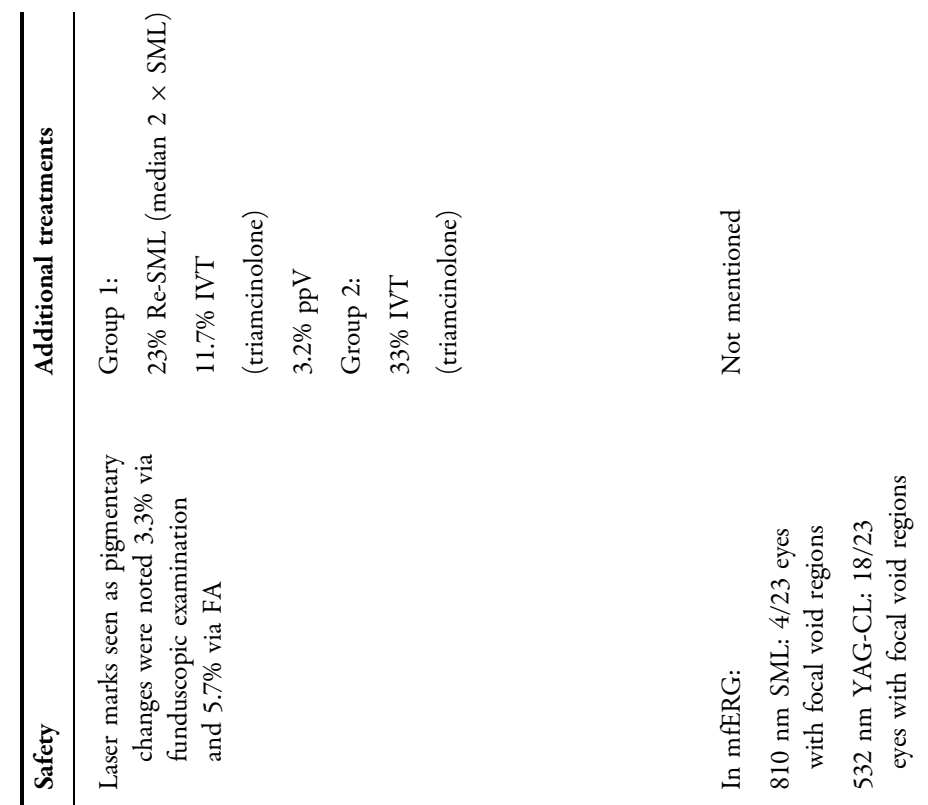

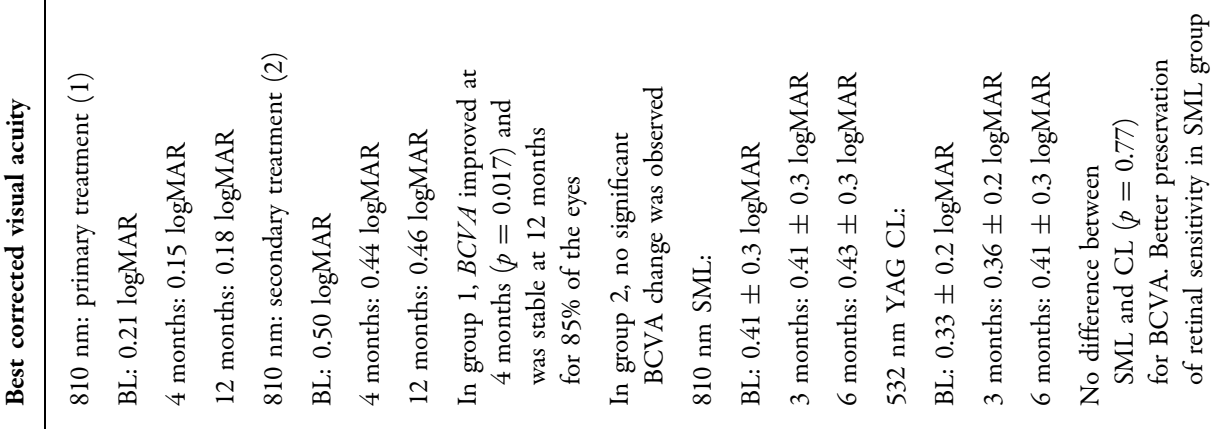

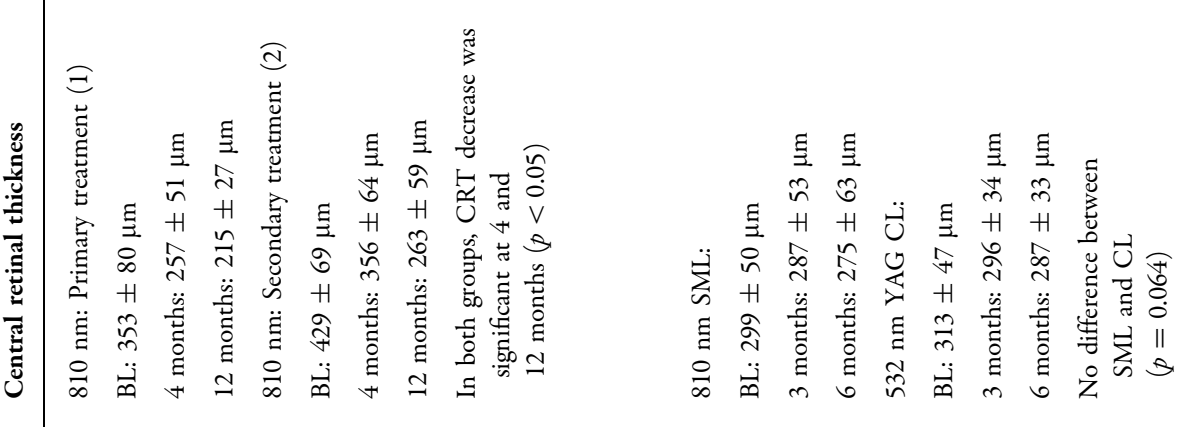

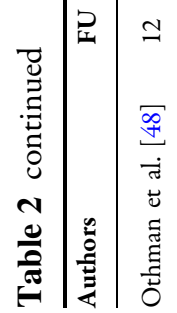

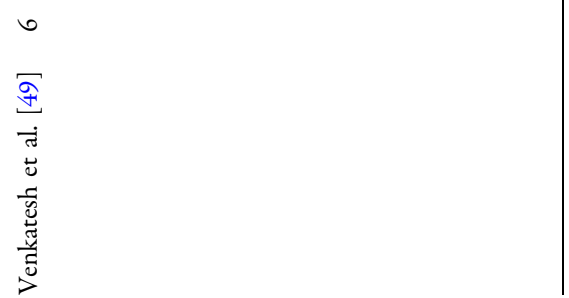




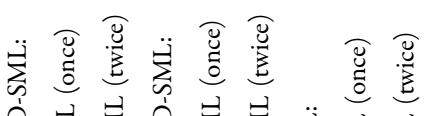

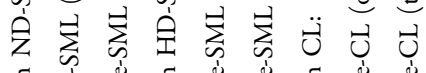

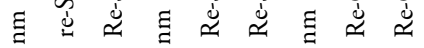

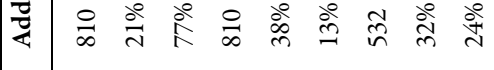

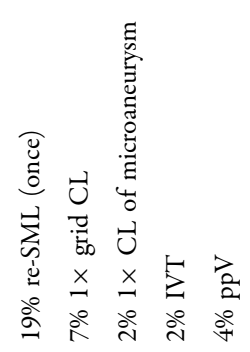

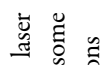

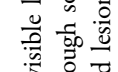

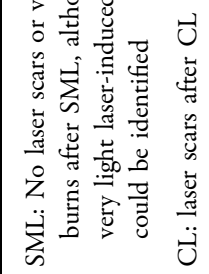

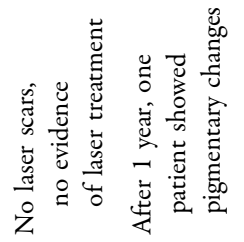

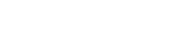

(2)

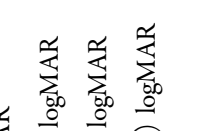

我这

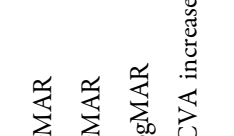

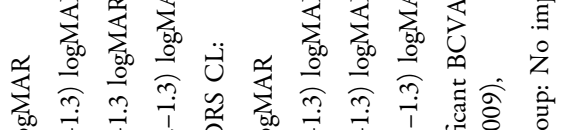

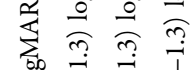

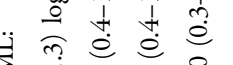

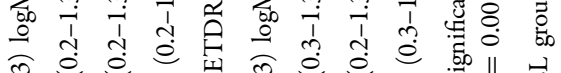

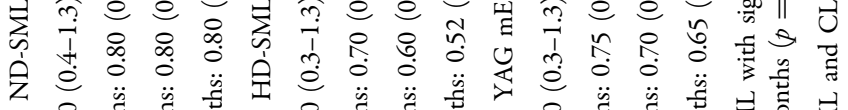

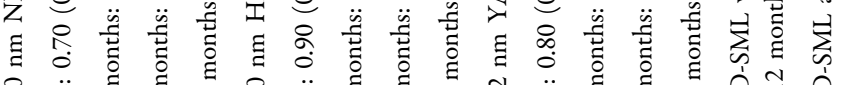

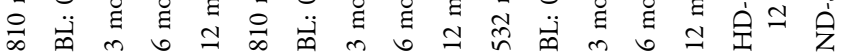

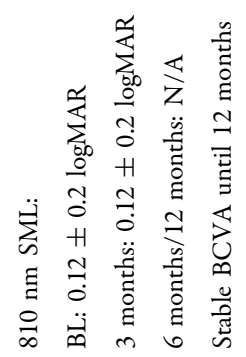

至至至

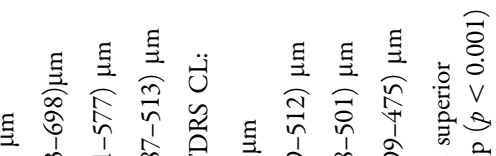

E

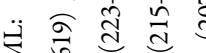

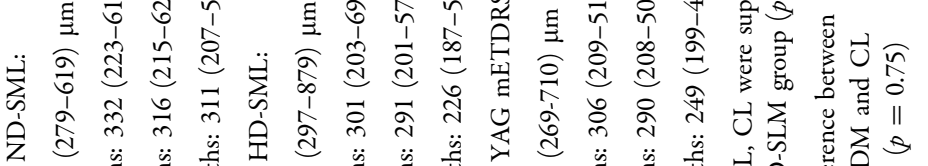

$\simeq$

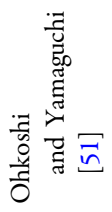




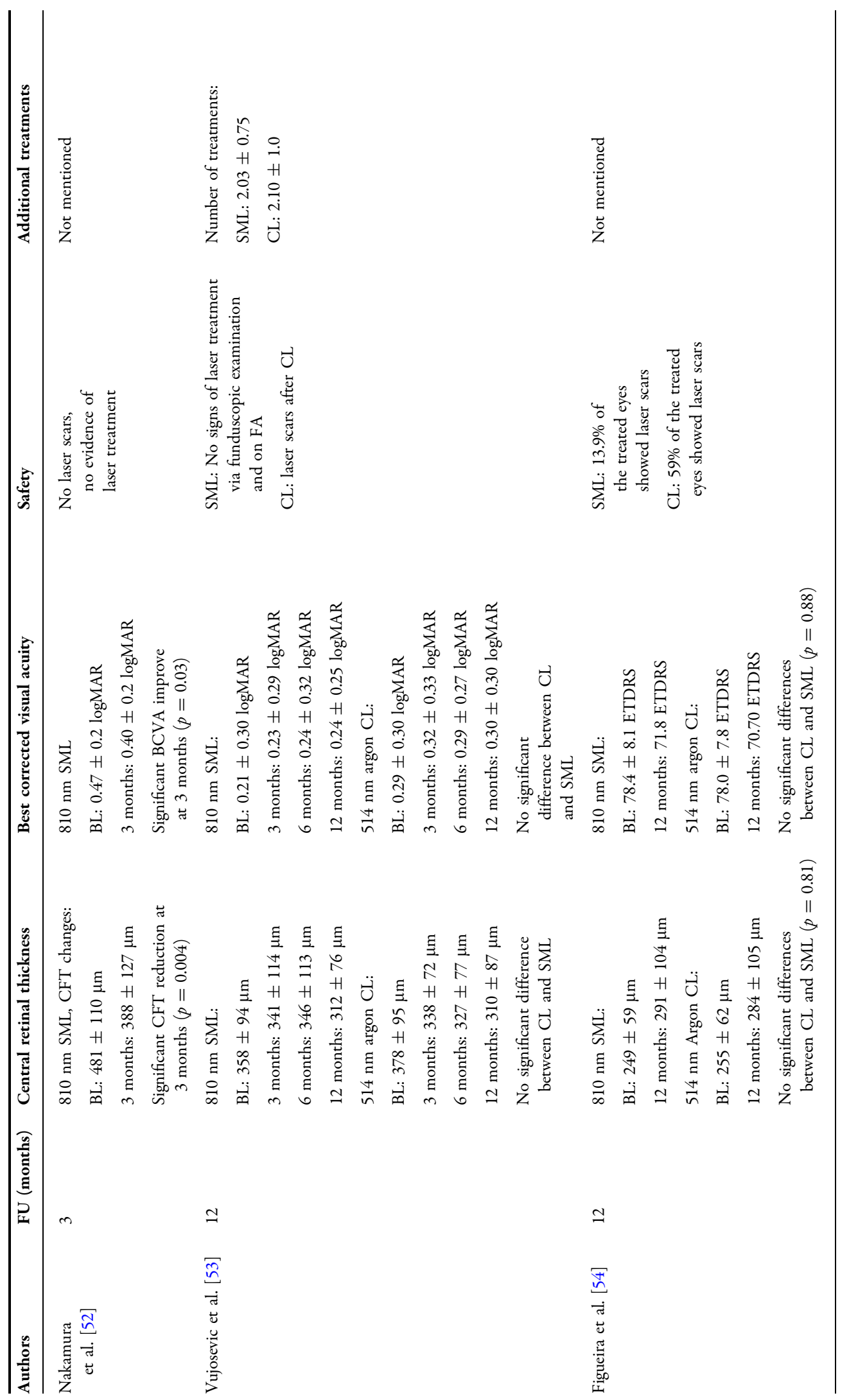




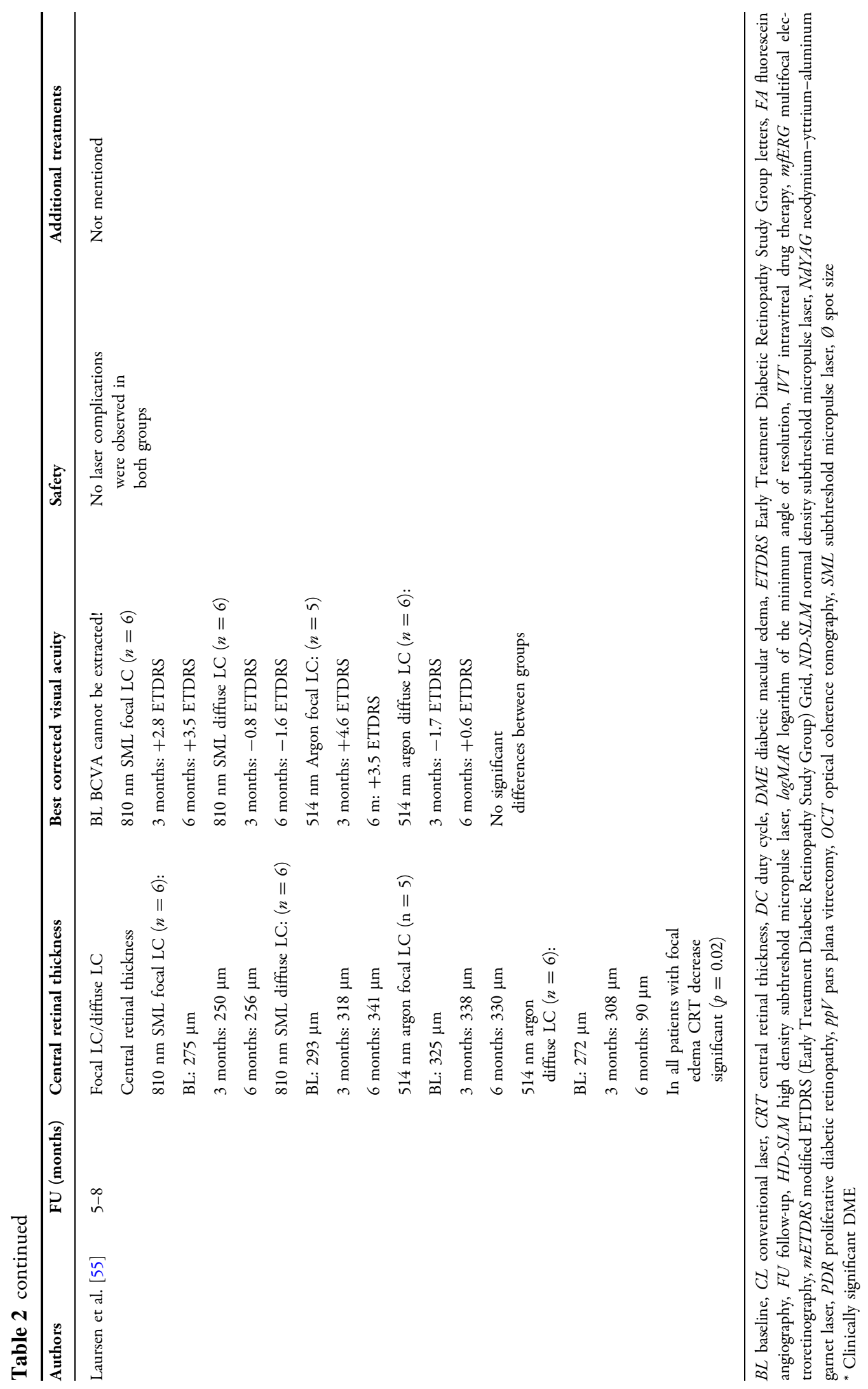


Table 3 Overview of the studies investigating subthreshold micropulse laser treatment for macular edema after branch retinal vein occlusion

\begin{tabular}{|c|c|c|c|c|c|}
\hline Authors & Year & Eyes & Inclusion criteria & $\begin{array}{l}\text { Laser type and } \\
\text { parameters }\end{array}$ & Study design \\
\hline $\begin{array}{l}\text { Parodi } \\
\text { et al. } \\
{[56]}\end{array}$ & 2015 & $\begin{array}{l}35 \text { eyes } \\
\text { Group 1: } \\
\text { SML: } n=18 \\
\text { Group 2: } \\
\text { IVT } \\
\text { Bevacizumab } \\
\text { (PRN after } 3 \\
\text { initial injections) } \\
n=17\end{array}$ & $\begin{array}{l}\text { ME to due BRVO } \\
\text { CFT }>250 \mu \mathrm{m} \\
\text { Without } \\
\text { non-perfusion } \geq 5 \\
\text { disc areas } \\
\text { All eyes were previously } \\
\text { treated with } \\
\text { conventional grid laser }\end{array}$ & $\begin{array}{l}\text { Iris Medical } \\
\text { OcuLight SLX } \\
810 \mathrm{~nm}, \varnothing 125 \mu \mathrm{m}, 15 \% \\
\text { DC, } 0.3 \mathrm{~s} \text {, power: } \\
\text { titration }\end{array}$ & $\begin{array}{l}\text { Prospective, randomized, } \\
\text { interventional }\end{array}$ \\
\hline $\begin{array}{l}\text { Inagaki } \\
\text { et al. } \\
\text { [57] }\end{array}$ & 2014 & $\begin{array}{l}32 \text { eyes } \\
\text { Group 1: } \\
\text { BCVA } \leq 20 / 40 \\
n=15 \\
\text { Group 2: } \\
\text { BCVA }>20 / 40 \\
n=17\end{array}$ & $\begin{array}{l}\text { ME due to BRVO } \\
\text { (ischemic/ } \\
\text { non-ischemic) } \\
\text { CRT }<600 \mu \mathrm{m} \\
\text { No prior macular } \\
\text { therapy (LC, IVT etc.) } \\
\text { within last } 6 \text { months }\end{array}$ & $\begin{array}{l}\text { Iris Medical } \\
\text { OcuLight SLX, } \\
810 \mathrm{~nm}, \varnothing 200 \mu \mathrm{m}, 15 \% \\
\text { DC, } 0.2 \text { or } 0.3 \mathrm{~s}, \\
\text { Power: } \\
750-1500 \mathrm{~mW}(90 \%) \\
\text { for } 0.2 \mathrm{~s} \text { or } \\
360-2000 \mathrm{~mW}(60 \%) \\
\text { for } 0.3 \mathrm{~s}\end{array}$ & $\begin{array}{l}\text { Retrospective, single-center, } \\
\text { nonrandomized, } \\
\text { interventional case series }\end{array}$ \\
\hline $\begin{array}{l}\text { Parodi } \\
\text { et al. } \\
{[58]}\end{array}$ & 2008 & $\begin{array}{l}24 \text { eyes } \\
\text { Group 1: } \\
\text { SML only } \\
\begin{array}{l}n=13 \\
\text { Group 2: } \\
\text { SML + IVT } \\
\text { Triamcinolone } \\
n=11\end{array}\end{array}$ & $\begin{array}{l}\text { ME due to BRVO } \\
\text { CRT }>212 \mu \mathrm{m} \\
\text { No prior laser } \\
\text { treatment } \\
\text { Without } \\
\text { non-perfusion } \geq 5 \text { disc } \\
\text { areas }\end{array}$ & $\begin{array}{l}\text { Iris Medical } \\
\text { OcuLight SLX, } \\
810 \mathrm{~nm} \\
\varnothing 125 \mu \mathrm{m} \\
15 \% \text { DC, } 0.3 \mathrm{~s} \\
\text { Power: titration }\end{array}$ & $\begin{array}{l}\text { Prospective randomized pilot } \\
\text { clinical trial }\end{array}$ \\
\hline $\begin{array}{l}\text { Parodi } \\
\text { et al. } \\
{[59]}\end{array}$ & 2006 & $\begin{array}{l}36 \text { eyes } \\
\text { Group 1: } \\
\text { SML grid } \\
n=17 \\
\text { Group 2: } \\
\text { Krypton grid } \\
n=19\end{array}$ & $\begin{array}{l}\text { ME due to BRVO } \\
\text { CRT }>210 \mu \mathrm{m} \\
\text { No prior laser } \\
\text { treatment } \\
\text { Without non-perfusion } \\
\geq 5 \text { disc areas }\end{array}$ & $\begin{array}{l}\text { Iris Medical } \\
\text { OcuLight SLX } 810 \mathrm{~nm} \\
\varnothing 125 \mu \mathrm{m}, 10 \% \mathrm{DC} \text {, } \\
0.2 \mathrm{~s} \text {, power: titration } \\
\text { Novus Omni Krypton } \\
\varnothing 100 \mu \mathrm{m}, 0.1 \mathrm{~s}\end{array}$ & $\begin{array}{l}\text { Prospective, randomized } \\
\text { clinical trial }\end{array}$ \\
\hline
\end{tabular}


Table 3 continued

\begin{tabular}{|c|c|c|c|c|c|}
\hline Authors & $\begin{array}{l}\text { FU } \\
\text { (months) }\end{array}$ & Central retinal thickness & Best corrected visual acuity & Safety & $\begin{array}{l}\text { Additional } \\
\text { treatments }\end{array}$ \\
\hline $\begin{array}{l}\text { Parodi et al. } \\
\text { [56] }\end{array}$ & 12 & $\begin{array}{l}\text { SML group (CFT): } \\
\text { BL: } 485.5 \mu \mathrm{m} \\
3 \text { months: } 472.0 \mu \mathrm{m} \\
6 \text { months: } 475.0 \mu \mathrm{m} \\
9 \text { months: } 475.0 \mu \mathrm{m} \\
12 \text { months: } 445.0 \mu \mathrm{m} \\
\text { IVT group }(\mathrm{CFT}): \\
\text { BL: } 484.2 \mu \mathrm{m} \\
3 \text { months: } 305.0 \mu \mathrm{m} \\
6 \text { months: } 266.0 \mu \mathrm{m} \\
9 \text { months: } 265.0 \mu \mathrm{m} \\
12 \text { months: } 271.0 \mu \mathrm{m} \\
\text { IVT group significantly better } \\
\text { ( } p=0.001)\end{array}$ & $\begin{array}{l}\text { SML group: } \\
\text { BL: } 0.92 \text { logMAR } \\
3 \text { months: } 0.89 \operatorname{logMAR} \\
6 \text { months: } 0.89 \log \text { MAR } \\
9 \text { months: } 0.94 \log \mathrm{MAR} \\
12 \text { months: } 0.99 \log \text { ARR } \\
\text { IVT group: } \\
\text { BL: } 0.94 \text { logMAR } \\
3 \text { months: } 0.88 \text { logMAR } \\
6 \text { months: } 0.88 \text { logMAR } \\
9 \text { months: } 0.85 \operatorname{logMAR} \\
12 \text { months: } 0.72 \text { logMAR } \\
\text { IVT group significantly better } \\
\text { ( } p=0.0085)\end{array}$ & $\begin{array}{c}\text { No laser } \\
\text { scars }\end{array}$ & Not mentioned \\
\hline $\begin{array}{l}\text { Inagaki et al. } \\
\text { [57] }\end{array}$ & 12 & $\begin{array}{l}\text { Group } 1 \text { : (BCVA } \leq 20 / 40 \\
\text { Snellen) } \\
\text { BL: } 409.3 \mu \mathrm{m} \\
1 \text { month: } 394.3 \mu \mathrm{m} \\
3 \text { months: } 371.3 \mu \mathrm{m} \\
6 \text { months: } 313.5 \mu \mathrm{m} \\
12 \text { months: } 303.5 \mu \mathrm{m} \\
\text { Group 2: (BCVA >20/40 } \\
\text { Snellen) } \\
\text { BL : } 373.3 \mu \mathrm{m} \\
1 \text { month: } 353.5 \mu \mathrm{m} \\
3 \text { months: } 313.1 \mu \mathrm{m} \\
6 \text { months: } 294.1 \mu \mathrm{m} \\
12 \text { months: } 320.1 \mu \mathrm{m} \\
\text { Significant CRT decrease at } 3 \text {, } \\
6 \text {, } \\
\text { and } 12 \text { months for both } \\
\text { groups. No } \\
\text { significant difference between } \\
\text { the } \\
\text { groups at any time point }\end{array}$ & $\begin{array}{l}\text { Group 1: (BCVA } \leq 20 / 40 \\
\text { Snellen) } \\
\text { BL: } 0.59 \text { logMAR } \\
1 \text { month: } 0.54 \log \mathrm{MAR} \\
3 \text { months: } 0.54 \log \mathrm{MAR} \\
6 \text { months: } 0.58 \log \mathrm{MAR} \\
12 \text { months: } 0.51 \log \mathrm{MAR} \\
\text { Group 2: (BCVA > 20/40 } \\
\text { Snellen) } \\
\text { BL: 0.13 logMAR } \\
1 \text { month: } 0.09 \log \mathrm{MAR} \\
3 \text { months: } 0.13 \log \mathrm{MAR} \\
6 \text { months: } 0.09 \log \mathrm{MAR} \\
12 \text { months: } 0.12 \log \mathrm{MAR}\end{array}$ & $\begin{array}{c}\text { No laser } \\
\text { scars }\end{array}$ & $\begin{array}{l}\text { Group 1: } \\
n=8(53.3 \%) \\
\text { Group 2: } \\
n=3(17.6 \%)\end{array}$ \\
\hline
\end{tabular}


Table 3 continued

\begin{tabular}{|c|c|c|c|c|c|}
\hline Authors & $\begin{array}{l}\text { FU } \\
\text { (months) }\end{array}$ & Central retinal thickness & Best corrected visual acuity & Safety & $\begin{array}{l}\text { Additional } \\
\text { treatments }\end{array}$ \\
\hline $\begin{array}{l}\text { Parodi } \\
\text { et al. } \\
\text { [58] }\end{array}$ & 12 & $\begin{array}{l}\text { SML only: } \\
\text { BL: } 429 \mu \mathrm{m} \\
3 \text { months: } 364 \mu \mathrm{m} \\
6 \text { months: } 320 \mu \mathrm{m} \\
9 \text { months: } 290 \mu \mathrm{m} \\
12 \text { months: } 278 \mu \mathrm{m} \\
\text { SML + IVT (triamcinolone): } \\
\text { BL: } 476 \mu \mathrm{m} \\
3 \text { months: } 269 \mu \mathrm{m} \\
6 \text { months: } 276 \mu \mathrm{m} \\
9 \text { months: } 260 \mu \mathrm{m} \\
12 \text { months: } 283 \mu \mathrm{m} \\
\text { Combined SML }+ \text { IVT showed } \\
\text { better response at } 3 \text { months } \\
\text { (p<0.001). No difference } \\
\text { between groups from 9th month } \\
\text { on }\end{array}$ & $\begin{array}{l}\text { SML only: } \\
\text { BL: } 0.76 \log \text { MAR } \\
3 \text { month: } 0.78 \log \mathrm{MAR} \\
6 \text { months: } 0.78 \log \mathrm{MAR} \\
9 \text { months: } 0.73 \log \mathrm{MAR} \\
12 \text { months: } 0.65 \log \mathrm{MAR} \\
\text { SML + IVT (triamcinolone): } \\
\text { BL: } 0.67 \text { logMAR } \\
3 \text { months: } 0.50 \text { logMAR } \\
6 \text { months: } 0.45 \log \mathrm{MAR} \\
9 \text { months: } 0.36 \log \mathrm{MAR} \\
12 \text { months: } 0.35 \text { logMAR } \\
\text { Combined SML + IVT showed } \\
\text { significant better response at } 9 \text { th } \\
\text { and } 12 \text { th months }(p<0.009, \\
p=0.011, \text { respectively) }\end{array}$ & $\begin{array}{l}\text { No } \\
\text { Laser } \\
\text { scars }\end{array}$ & $\begin{array}{l}\text { Not } \\
\text { mentioned }\end{array}$ \\
\hline $\begin{array}{l}\text { Parodi } \\
\text { et al. } \\
\text { [59] }\end{array}$ & 24 & $\begin{array}{l}\text { SML grid: } \\
\text { BL: } 480 \mu \mathrm{m} \\
6 \text { months: } 457 \mu \mathrm{m} \\
12 \text { months: } 217 \mu \mathrm{m} \\
18 \text { months: } 215 \mu \mathrm{m} \\
24 \text { months: } 208 \mu \mathrm{m} \\
\text { Krypton grid: } \\
\text { BL: } 454 \mu \mathrm{m} \\
6 \text { months: } 252 \mu \mathrm{m} \\
12 \text { months: } 226 \mu \mathrm{m} \\
18 \text { months: } 229 \mu \mathrm{m} \\
24 \text { months: } 217 \mu \mathrm{m} \\
\text { Krypton showed better response at } \\
3 \text { months and } 6 \text { months } \\
\text { (p<0.001). SML showed better } \\
\text { response from 12th month on } \\
(p<0.001)\end{array}$ & $\begin{array}{l}\text { SML grid: } \\
\text { BL: } 0.70 \text { logMAR } \\
6 \text { months: } 0.70 \operatorname{logMAR} \\
9 \text { months: } 0.55 \operatorname{logMAR} \\
12 \text { months: } 0.51 \log \mathrm{MAR} \\
24 \text { months: } 0.49 \log \mathrm{MAR} \\
\text { Krypton grid: } \\
\text { BL: } 0.69 \text { logMAR } \\
6 \text { months: } 0.60 \operatorname{logMAR} \\
9 \text { months: } 0.58 \operatorname{logMAR} \\
12 \text { months } 0.57 \text { logMAR } \\
24 \text { m: } 0.56 \text { logMAR } \\
\text { No statistical difference between } \\
\text { groups }\end{array}$ & $\begin{array}{l}\text { No } \\
\text { laser } \\
\text { scars } \\
\text { after } \\
\text { SML }\end{array}$ & $\begin{array}{l}\text { Not } \\
\text { mentioned }\end{array}$ \\
\hline
\end{tabular}

$B R V O$ branch retinal vein occlusion, $B L$ baseline, $C F T$ central foveal thickness, $C R T$ central retinal thickness, $D C$ duty cycle, $F A$ fluorescein angiography, $I V T$ intravitreal drug therapy, $\log M A R$ logarithm of the minimum angle of resolution, $M E$ macular edema, $P R N$ pro re nata, $S M L$ subthreshold micropulse laser 
Table 4 Treatment outcome after SML, PDT, observation and conventional laser for CSC, DME, and BRVO

\begin{tabular}{llcc}
\hline & Treatment & Change in CRT $(\boldsymbol{\mu m})$ & Change in BCVA (ETDRS letters) \\
\hline CSC & SML & $-131(\text { range }-69.7 \text { to }-204)^{\mathrm{a}}$ & $6.34(\text { range }-15 \text { to } 20)^{\mathrm{d}}$ \\
& PDT & $-85(\text { range }-76 \text { to }-109.8)^{\mathrm{b}}$ & $3.87(\text { range } 2 \text { to } 8.5)^{\mathrm{b}}$ \\
\multirow{2}{*}{ DME } & Observation & $-25(\text { range } 26 \text { to }-89)^{\mathrm{c}}$ & $0.67(\text { range }-2.1 \text { to } 2.5)^{\mathrm{c}}$ \\
& SML & $-74.9(\text { range }-138 \text { to } 48)^{\mathrm{e}}$ & $1.26(\text { range }-6.6 \text { to } 19)^{\mathrm{e}}$ \\
BRVO & Conventional laser & $-43.6(\text { range }-145 \text { to } 28.7)^{\mathrm{f}}$ & $-0.29(\text { range }-7.3 \text { to } 7.5)^{\mathrm{f}}$ \\
\hline
\end{tabular}

$C S C$ central serous chorioretinopathy, DME diabetic macular edema, $B R V O$ branch retinal vein occlusion, $B C V A$ best corrected visual acuity, $C R T$ central retinal thickness, ETDRS Early Treatment Diabetic Retinopathy Study Group letters, $P D T$ photodynamic therapy, $S M L$ subthreshold micropulse laser

a 199 patients from 11 studies, 7 studies excluded from the calculations, one due to prior PDT treatment [37], six due to absence of information about the CRT

b 100 patients from 3 studies

c 49 patients from 3 studies

d 216 patients from 14 studies, two studies excluded due to prior PDT [37, 41], two due to absence of information about the concrete BCVA $[28,31]$

e 613 patients from 11 studies

e 195 patients from 7 studies

f 80 patients from 3 studies, one study excluded from the calculation due to prior conventional laser treatment [56]

OCT (SDOCT). A complete resolution of SRF in SDOCT was defined as a complete treatment response. Two studies measured the leakage activity in FA as a parameter for treatment response [32, 35]. For simplicity reasons we do not distinguish between the different definitions for treatment response in our calculations. Few studies did not mention the amount of patients with treatment response. If we were able to work out the treatment response from the data shown in the paper, we quote the response; otherwise the studies were excluded from the calculations $[33,38]$. One case report was excluded from the calculation because of prior bevacizumab treatment [39], and two studies were excluded since they included patients with prior PDT [37, 41]. Few studies mentioned only the response or the complete response, and those studies were included in the calculations.

We included 191 patients from 12 studies for the calculations of the treatment response and 176 patients from 11 studies for the complete response. A total of $156(79.6 \%)$ of the 191 patients showed a treatment response at the last mentioned follow-up: $112(63.6 \%)$ of the 176 patients had a complete resolution of SRF. Only two studies showed data concerning the improvement rate in an untreated control group: a complete resolution of SRF was seen in $2(8 \%)$ out of 26 eyes at the last follow-up and a reduction in SRF in 7 (39\%) out of 18 eyes.

Four studies had a control group consisting of patients receiving PDT treatment (half dose PDT in three studies and half fluence PDT in one). The treatment response could be calculated from 100 patients in three studies and the complete treatment response from 135 patients in three studies. A total of $64(64 \%)$ of the 100 patients responded to PDT and 62 (46\%) of 135 patients showed complete response.

\section{Safety}

The majority of studies described no visible retinal changes after the micropulse laser treatment. In six patients from two studies [30, 39] pigmentary changes at the level of the RPE were seen after SML but without any visual implications for the patients. Complications like scar 
formation, visible laser burns, or $\mathrm{CNV}$ did not occur.

\section{DIABETIC MACULAR EDEMA (DME)}

DME is a frequent complication of diabetic retinopathy (DR) and the most common cause of visual impairment in patients with DR [5]. Since the ETDRS trial [1, 73] showed that laser photocoagulation reduced the risk of moderate visual loss by $50 \%$ in eyes with clinically significant macular edema, laser photocoagulation became the standard therapy for DME for many years. Depending on the kind of edema, the treatment pattern can be selected: a focal photocoagulation for localized areas of leakage and a grid pattern for a diffuse macular edema. Continuous-wave photocoagulation comes with potential side effects like epiretinal fibrosis, CNV, and enlargement of laser scars $[7,8,74]$. Table 3 shows only the prospective studies investigating micropulse laser treatment for diabetic macular edema. A total of 613 patients from 11 studies were included in the calculations. The inclusion and exclusion criteria varied between studies; some did not allow prior treatment at all, most of them only excluded patients with treatment in the prior 3-6 months. All listed studies were included in the calculations for change in CRT and BCVA. Seven studies had a control group consisting of 195 patients treated with conventional laser. The same calculations were performed for those studies.

Table 4 displays the treatment outcome after SML and conventional laser for DME.

\section{Safety}

In the majority of studies no laser scars occurred after SML. Four studies reported scar formation or pigmentary changes in a small amount of eyes after SML treatment $[48,50,51,54]$. Retinal changes were only observed in eyes treated with duty cycles of $15 \%$; lower duty cycles did not lead to scar formation in the listed studies.

Venkatesh [49] et al. reported focal void regions in multifocal electroretinogram in 4 out of 23 eyes after SML treatment with 10\% duty cycle compared to 18 out of 23 eyes after conventional laser.

\section{MACULAR EDEMA DUE TO RETINAL VEIN OCCLUSION (RVO)}

Macular edema is a common complication of branch RVO (BRVO) [75]. Grid laser photocoagulation reduces the visual acuity loss after BRVO with macular edema [75]. Parodi et al. [59] reported a similar outcome in visual acuity improvement and resolution of macular edema after SML treatment compared to conventional laser, but without retinal changes after SML. Table 3 summarizes studies investigating SML treatment for macular edema after BRVO. In total 80 patients from three studies could be included in the calculations, and one study was excluded because of prior conventional laser treatment [56]. As a result of the small number of studies and the variety in control groups (bevacizumab, SML + triamcinolone, conventional laser), the control groups were not separately analyzed. Only one study [48] had a control group where patients were treated with anti-VEGF agents, the current standard therapy for macular edema due to BRVO.

Table 4 presents the treatment outcome after SML for macular edema after BRVO.

\section{Safety}

No study described complications like scar formation, visible laser burns, or CNV.

\section{PROBLEMS AND CHALLENGES OF SML TREATMENT}

Although the majority of the studies showed some efficacy of the SML treatment for CSC, DME, or BRVO, the treatment parameter differed significantly between the individual studies. No study compared the outcome of SML with different treatment parameters like higher or lower duty cycle. Concerning the treatment power, most authors titrated the power individually for each patient, but the 
path was not consistent. The titration is probably the most challenging part of the SML treatment. Since the laser surgeon did not see an effect of the treatment, there is a high risk of undertreatment and treatment failure accordingly. A solution to this problem could be to use fixed laser parameters with the same power for all patients. But so far there is not enough published data to choose the best treatment power and to evaluate the safety and the treatment success of subthreshold micropulse treatment with fixed parameters. For the future, controlled trials comparing treatment outcome and safety of individual titrated SML treatment and SML treatment with fixed parameters would be desirable. Those studies should include safety follow-up with multimodal imaging including autofluorescence, OCT, and fundus photographies as well functional follow-up with microperimetry or multifocal electroretinogram.

\section{CONCLUSION}

For CSC, the presented studies showed a higher efficacy of the micropulse laser treatment for both morphology and visual function in comparison to no treatment or PDT. The decrease in CRT was highest after SML $(-131 \mu \mathrm{m})$, followed by PDT $(-85 \mu \mathrm{m})$ and the no-treatment group $(-25 \mu \mathrm{m})$. Moreover, $64 \%$ of patients showed no SRF after SML compared to $46 \%$ after PDT and $8 \%$ after observation.

No study reported any complications after up to five SML treatment sessions, so even an early treatment could be considered for potentially better results. Chen et al. [29] showed that the SML treatment outcome was best in patients with source leakage without RPE atrophy. The investigated literature did not allow an evaluation of the best treatment parameter or the best laser wavelength.

Regarding the treatment of DME, the investigated studies showed efficacy also in morphology and function. The decrease in CRT and increase in BCVA after SML $(-74.9 \mu \mathrm{m}$ and +1.26 ETDRS letters) was better than after conventional laser $(-43.6 \mu \mathrm{m}$ and -0.29 ETDRS letters), but no study had a control group in which patients were treated with anti-VEGF agents. After the RISE and RIDE studies [76] and the approval of ranibizumab for the treatment of DME, anti-VEGF agents became the standard treatment for DME. Without any trial, comparing SML treatment with anti-VEGF agents, we do not know when SML treatment could be an alternative first-line treatment for DME. Nevertheless, SML might be an option in patients not responding sufficiently to, or who are not able to follow an anti-VEGF therapy (e.g., high costs, compliance problems due to frequent visits for the injections and ophthalmological controls). Chen et al. [77] had come to a similar result in their meta-analysis of randomized controlled trials comparing subthreshold micropulse diode laser photocoagulation and conventional laser. They reported a significantly better visual acuity and a similar decrease in CRT after SML compared to conventional laser. They underline the advantage of the SML treatment in terms of the affordability compared to the cost-intensive anti-VEGF therapy.

On the subject of macular edema after BRVO, SML treatment shows some efficacy as well. But in comparison to the current standard treatment, intravitreal anti-VEGF, SML was inferior to intravitreal bevacizumab [56]. However, similar to DME, SML treatment could be an option for adjunct treatment for selected patients.

In summary, in all three indications micropulse laser is an efficacious and safe treatment option. Owing to its higher efficacy and the excellent safety profile compared to PDT, it could become the first-line therapy in CSC, potentially even in acute cases.

\section{ACKNOWLEDGEMENTS}

No funding or sponsorship was received for this study or publication of this article. All named authors meet the International Committee of Medical Journal Editors (ICMJE) criteria for authorship for this manuscript, take responsibility for the integrity of the work as a whole, and have given final approval for the version to be published. 
Disclosures. Paula Scholz received a speaker honorarium from Quantel Medical. Sascha Fauser received a speaker honorarium from Quantel Medical. Lebriz Altay has nothing to disclose.

Compliance With Ethics Guidelines. This article is based on previously conducted studies and does not involve any new studies of human or animal subjects performed by any of the authors.

Data Availability. The datasets generated and analyzed during the current study are available from the corresponding author on reasonable request.

Open Access. This article is distributed under the terms of the Creative Commons Attribution-NonCommercial 4.0 International License (http://creativecommons.org/licenses/ by-nc/4.0/), which permits any noncommercial use, distribution, and reproduction in any medium, provided you give appropriate credit to the original author(s) and the source, provide a link to the Creative Commons license, and indicate if changes were made.

\section{REFERENCES}

1. Early Treatment Diabetic Retinopathy Study Research Group. Photocoagulation for diabetic macular edema. Early treatment diabetic retinopathy study report number 1. Arch Ophthalmol. 1985;103:1796-806.

2. Robertson DM, Ilstrup D. Direct, indirect, and sham laser photocoagulation in the management of central serous chorioretinopathy. Am J Ophthalmol. 1983;95(4):457-66.

3. Ficker L, Vafidis G, While A, Leaver P. Long-term follow-up of a prospective trial of argon laser photocoagulation in the treatment of central serous retinopathy. Br J Ophthalmol. 1988;72(11):829-34.

4. The Branch Vein Occlusion Study Group. Argon laser photocoagulation for macular edema in branch vein occlusion. Am J Ophthalmol. 1984;98(3):271-82.

5. The Diabetic Retinopathy Study Research Group. Preliminary report on effects of photocoagulation therapy. Am J Ophthalmol. 1976;81(4):383-96.
6. Ulbig MR, Arden GB, Hamilton AP. Color contrast sensitivity and pattern electroretinographic findings after diode and argon laser photocoagulation in diabetic retinopathy. Am J Ophthalmol. 1994;117(5):583-8.

7. Lewen RM. Subretinal neovascularization complicating laser photocoagulation of diabetic maculopathy. Ophthal Surg Lasers Imaging Retina. 1988;19(10):734-7.

8. Smith CW, Guyer DR, D'Amico DJ. Subretinal fibrosis after laser photocoagulation for diabetic macular edema. Am J Ophthalmol. 1992;113(6):652-6.

9. Seiberth V, Alexandridis E, Feng W. Function of the diabetic retina after panretinal argon laser coagulation. Graefes Arch Clin Exp Ophthalmol. 1987;225(6):385-90.

10. Pearson A, Tanner V, Keightley S, Casswell A. What effect does laser photocoagulation have on driving visual fields in diabetics? Eye (Lond). 1998;12(1):64-8.

11. Roider J. Laser treatment of retinal diseases by subthreshold laser effects. Semin Ophthalmol. 2009;14(19-26).

12. Bresnick GH. Diabetic maculopathy: a critical review highlighting diffuse macular edema. Ophthalmology. 1983;90(11):1301-17.

13. Wilson AS, Hobbs BG, Shen W-Y, et al. Argon laser photocoagulation-induced modification of gene expression in the retina. Invest Ophthalmol Vis Sci. 2003;44(4):1426-34.

14. Dorin G. Evolution of retinal laser therapy: minimum intensity photocoagulation (MIP). Can the laser heal the retina without harming it? Semin Ophthalmol. 2004;19(1-2):62-8.

15. Inagaki $\mathrm{K}$, Shuo $\mathrm{T}$, Katakura $\mathrm{K}$, Ebihara $\mathrm{N}$, Murakami A, Ohkoshi K. Sublethal photothermal stimulation with a micropulse laser induces heat shock protein expression in ARPE-19 cells. J Ophthalmol. 2015;2015:729792.

16. Lanzetta P, Dorin G, Pirracchio A, Bandello F. Theoretical bases of non-ophthalmoscopically visible endpoint photocoagulation. Semin Ophthalmol. 2001;16(1):8-11.

17. Anderson RR, Parrish JA. Selective photothermolysis: precise microsurgery by selective absorption of pulsed radiation. Science. 1983;220(4596):524-7.

18. Roider J, Hillenkamp F, Flotte T, Birngruber R. Microphotocoagulation: selective effects of 
repetitive short laser pulses. Proc Natl Acad Sci. 1993;90(18):8643-7.

19. Roider J, Michaud NA, Flotte TJ, Birngruber R. Response of the retinal pigment epithelium to selective photocoagulation. Arch Ophthalmol. 1992;110(12):1786-92.

20. Dorin G, editor. Subthreshold and micropulse diode laser photocoagulation. Seminars in Ophthalmology. 2003;18(3):147-53.

21. McHugh J, Marshall J, Ffytche T, Hamilton A, Raven A. Macular photocagulation of human retina with a diode laser: a comparative histopathological study. Lasers Light Ophthalmol. 1990;3(1):11-28.

22. Vogel A, Birngruber R. Temperature profiles in human retina and choroid during laser coagulation with different wavelengths ranging from 514 to $810 \mathrm{~nm}$. Lasers Light Ophthalmol. 1992;5(1):9-16.

23. Peyman GA, Raichand M, Zeimer RC. Ocular effects of various laser wavelengths. Surv Ophthalmol. 1984;28(5):391-404.

24. Friberg TR, Karatza EC. The treatment of macular disease using a micropulsed and continuous wave 810-nm diode laser. Ophthalmology. 1997;104(12):2030-8.

25. Friberg TR, Venkatesh MdS. Alteration of pulse configuration affects the pain response during diode laser photocoagulation. Lasers Surg Med. 1995;16(4):380-3.

26. Mainster MA. Wavelength selection in macular photocoagulation: tissue optics, thermal effects, and laser systems. Ophthalmology. 1986;93(7):952-8.

27. Ricci F, Missiroli F, Cerulli L. Indocyanine green dye-enhanced micropulsed diode laser: a novel approach to subthreshold RPE treatment in a case of central serous chorioretinopathy. Eur J Ophthalmol. 2004;14(1):74-82.

28. Ricci F, Missiroli F, Regine F, Grossi M, Dorin G. Indocyanine green enhanced subthreshold diodelaser micropulse photocoagulation treatment of chronic central serous chorioretinopathy. Graefes Arch Clin Exp Ophthalmol. 2009;247(5):597-607.

29. Chen S-N, Hwang J-F, Tseng L-F, Lin C-J. Subthreshold diode micropulse photocoagulation for the treatment of chronic central serous chorioretinopathy with juxtafoveal leakage. Ophthalmology. 2008;115(12):2229-34.

30. Lanzetta P, Furlan F, Morgante L, Veritti D, Bandello F. Nonvisible subthreshold micropulse diode laser $(810 \mathrm{~nm})$ treatment of central serous chorioretinopathy. A pilot study. Eur J Ophthalmol. 2007;18(6):934-40.

31. Gupta B, Elagouz M, McHugh D, Chong V, Sivaprasad S. Micropulse diode laser photocoagulation for central serous chorio-retinopathy. Clin Exp Ophthalmol. 2009;37(8):801-5.

32. Koss M, Beger I, Koch F. Subthreshold diode laser micropulse photocoagulation versus intravitreal injections of bevacizumab in the treatment of central serous chorioretinopathy. Eye (Lond). 2011;26(2):307-14.

33. Roisman L, Magalhães FP, Lavinsky D, et al. Micropulse diode laser treatment for chronic central serous chorioretinopathy: a randomized pilot trial. Ophthalmic Surg Lasers Imaging Retina. 2013;44(5):465-70.

34. Malik KJ, Sampat KM, Mansouri A, Steiner JN, Glaser BM. Low-intensity/high-density subthreshold micropulse diode laser for chronic central serous chorioretinopathy. Retina. 2015;35(3):532-6.

35. Kretz FT, Beger I, Koch F, Nowomiejska K, Auffarth GU, Koss MJ. Randomized clinical trial to compare micropulse photocoagulation versus half-dose verteporfin photodynamic therapy in the treatment of central serous chorioretinopathy. Ophthalmic Surg Lasers Imaging Retina. 2015;46(8):837-43.

36. Elhamid AHA. Subthreshold micropulse yellow laser treatment for nonresolving central serous chorioretinopathy. Clin Ophthalmol. 2015;9:2277.

37. Scholz P, Ersoy L, Boon CJ, Fauser S. Subthreshold micropulse laser $(577 \mathrm{~nm})$ treatment in chronic central serous chorioretinopathy. Ophthalmologica. 2015;234(4):189-94.

38. Kim JY, Park HS, Kim SY. Short-term efficacy of subthreshold micropulse yellow laser (577-nm) photocoagulation for chronic central serous chorioretinopathy. Graefes Arch Clin Exp Ophthalmol. 2015;253(12):2129-35.

39. Gawęcki M. Increase in central retinal edema after subthreshold diode micropulse laser treatment of chronic central serous chorioretinopathy. Case Rep Ophthalmol Med. 2015;2015:813414.

40. Yadav N, Jayadev C, Mohan A, et al. Subthreshold micropulse yellow laser $(577 \mathrm{~nm})$ in chronic central serous chorioretinopathy: safety profile and treatment outcome. Eye (Lond). 2015;29(2):258-64.

41. Breukink MB, Mohr JK, Ossewaarde-van Norel A, et al. Half-dose photodynamic therapy followed by diode micropulse laser therapy as treatment for chronic central serous chorioretinopathy: evaluation of a prospective treatment protocol. Acta Ophthalmol. 2016;94(2):187-97. 
42. Özmert E, Demirel S, Yanık Ö, Batığlu F. Low-fluence photodynamic therapy versus subthreshold micropulse yellow wavelength laser in the treatment of chronic central serous chorioretinopathy. J Ophthalmol. 2016;2016:3513794.

43. Ambiya V, Goud A, Mathai A, Rani PK, Chhablani J. Microsecond yellow laser for subfoveal leaks in central serous chorioretinopathy. Clin Ophthalmol (Auckland, NZ). 2016;10:1513.

44. Scholz P, Altay L, Fauser S. Comparison of subthreshold micropulse laser $(577 \mathrm{~nm})$ treatment and half-dose photodynamic therapy in patients with chronic central serous chorioretinopathy. Eye (Lond). 2016;30(10):1371-7.

45. Fazel F, Bagheri M, Golabchi K, Ardakani HJ. Comparison of subthreshold diode laser micropulse therapy versus conventional photocoagulation laser therapy as primary treatment of diabetic macular edema. J Curr Ophthalmol. 2016;28(4):206-11.

46. Inagaki $\mathrm{K}$, Ohkoshi $\mathrm{K}$, Ohde $\mathrm{S}$, Deshpande GA, Ebihara N, Murakami A. Comparative efficacy of pure yellow (577-nm) and 810-nm subthreshold micropulse laser photocoagulation combined with yellow (561-577-nm) direct photocoagulation for diabetic macular edema. Jpn J Ophthalmol. 2015;59(1):21-8.

47. Vujosevic S, Martini F, Longhin E, Convento E, Cavarzeran F, Midena E. Subthreshold micropulse yellow laser versus subthreshold micropulse infrared laser in center-involving diabetic macular edema: morphologic and functional safety. Retina. 2015;35(8):1594-603.

48. Othman IS, Eissa SA, Kotb MS, Sadek SH. Subthreshold diode-laser micropulse photocoagulation as a primary and secondary line of treatment in management of diabetic macular edema. Clin Ophthalmol. 2014;8:653.

49. Venkatesh P, Ramanjulu R, Azad R, Vohra R, Garg S. Subthreshold micropulse diode laser and double frequency neodymium:YAG laser in treatment of diabetic macular edema: a prospective, randomized study using multifocal electroretinography. Photomed Laser Surg. 2011;29(11):727-33.

50. Lavinsky D, Cardillo JA, Melo LA, Dare A, Farah ME, Belfort R. Randomized clinical trial evaluating mETDRS versus normal or high-density micropulse photocoagulation for diabetic macular edema. Invest Ophthalmol Vis Sci. 2011;52(7):4314-23.

51. Ohkoshi K, Yamaguchi T. Subthreshold micropulse diode laser photocoagulation for diabetic macular edema in Japanese patients. Am J Ophthalmol. 2010;149(1):133-9.
52. Nakamura Y, Mitamura Y, Ogata K, Arai M, Takatsuna Y, Yamamoto S. Functional and morphological changes of macula after subthreshold micropulse diode laser photocoagulation for diabetic macular oedema. Eye (Lond). 2010;24(5):784-8.

53. Vujosevic S, Bottega E, Casciano M, Pilotto E, Convento E, Midena E. Microperimetry and fundus autofluorescence in diabetic macular edema: subthreshold micropulse diode laser versus modified early treatment diabetic retinopathy study laser photocoagulation. Retina. 2010;30(6): 908-16.

54. Figueira J, Khan J, Nunes S, et al. Prospective randomised controlled trial comparing sub-threshold micropulse diode laser photocoagulation and conventional green laser for clinically significant diabetic macular oedema. $\mathrm{Br} \mathrm{J}$ Ophthalmol. 2009;93(10):1341-4.

55. Laursen M, Moeller F, Sander B, Sjoelie A. Subthreshold micropulse diode laser treatment in diabetic macular oedema. $\mathrm{Br} \mathrm{J}$ Ophthalmol. 2004;88(9):1173-9.

56. Parodi MB, Iacono P, Bandello F. Subthreshold grid laser versus intravitreal bevacizumab as second-line therapy for macular edema in branch retinal vein occlusion recurring after conventional grid laser treatment. Graefes Arch Clin Exp Ophthalmol. 2015;253(10):1647-51.

57. Inagaki $\mathrm{K}$, Ohkoshi $\mathrm{K}$, Ohde $\mathrm{S}$, Deshpande GA, Ebihara N, Murakami A. Subthreshold micropulse photocoagulation for persistent macular edema secondary to branch retinal vein occlusion including best-corrected visual acuity greater than 20/40. J Ophthalmol. 2014;2014:251257.

58. Parodi $\mathrm{MB}$, Iacono $\mathrm{P}$, Ravalico G. Intravitreal triamcinolone acetonide combined with subthreshold grid laser treatment for macular oedema in branch retinal vein occlusion: a pilot study. Br J Ophthalmol. 2008;92(8):1046-50.

59. Parodi MB, Spasse S, Iacono P, Di Stefano G, Canziani T, Ravalico G. Subthreshold grid laser treatment of macular edema secondary to branch retinal vein occlusion with micropulse infrared $(810$ nanometer) diode laser. Ophthalmology. 2006;113(12):2237-42.

60. Gregori NZ, Feuer W, Rosenfeld PJ. Novel method for analyzing snellen visual acuity measurements. Retina. 2010;30(7):1046-50.

61. Gass J. Pathogenesis of disciform detachment of the neuroepithelium. II. Idiopathic central serous choroidopathy. Am J Ophthalmol. 1967;63:587-615. 
62. Gilbert CM, Owens SL, Smith PD, Fine SL. Long-term follow-up of central serous chorioretinopathy. Br J Ophthalmol. 1984;68(11):815-20.

63. Fok AC, Chan PP, Lam DS, Lai TY. Risk factors for recurrence of serous macular detachment in untreated patients with central serous chorioretinopathy. Ophthalmic Res. 2011;46(3):160-3.

64. Wang MS, Sander B, Larsen M. Retinal atrophy in idiopathic central serous chorioretinopathy. Am J Ophthalmol. 2002;133(6):787-93.

65. Schatz H, Yannuzzi LA, Gitter KA. Subretinal neovascularization following argon laser photocoagulation treatment for central serous chorioretinopathy: complication or misdiagnosis? Retina. 2012;32:OP-893-906.

66. Schatz H, Madeira D, McDonald HR, Johnson RN. Progressive enlargement of laser scars following grid laser photocoagulation for diffuse diabetic macular edema. Arch Ophthalmol. 1991;109(11):1549-51.

67. Khosla P, Rana S, Tewari H, Azad R, Talwar D. Evaluation of visual function following argon laser photocoagulation in central serous retinopathy. Ophthalmic Surg Lasers. 1997;28(8):693-7.

68. Piccolino FC, Eandi CM, Ventre L, de la Longrais RCR, Grignolo FM. Photodynamic therapy for chronic central serous chorioretinopathy. Retina. 2003;23(6):752-63.

69. Chan W, Lam D, Lai T, Tam B, Liu D, Chan C. Choroidal vascular remodelling in central serous chorioretinopathy after indocyanine green guided photodynamic therapy with verteporfin: a novel treatment at the primary disease level. Br J Ophthalmol. 2003;87(12):1453-8.
70. Colucciello M. Choroidal neovascularization complicating photodynamic therapy for central serous retinopathy. Retina. 2006;26(2):239-42.

71. Lai TY, Chan W-M, Lam DS. Transient reduction in retinal function revealed by multifocal electroretinogram after photodynamic therapy. Am J Ophthalmol. 2004;137(5):826-33.

72. Bandello F, Lanzetta P, Furlan F, Polito A. Non visible subthreshold micropulse diode laser treatment of idiopathic central serous chorioretinopathy. A pilot study. Investig Ophtalmol Vis Sci. 2003;44(5):4858.

73. Early Treatment Diabetic Retinopathy Study Research Group. Early photocoagulation for diabetic retinopathy: ETDRS report number 9. Ophthalmology. 1991;98(5):766-85.

74. Morgan CM, Schatz H. Atrophic creep of the retinal pigment epithelium after focal macular photocoagulation. Ophthalmology. 1989;96(1):96-103.

75. Greer D, Constable I, Cooper R. Macular oedema and retinal branch vein occlusion. Aust J Opthalmol. 1980;8(3):207-9.

76. Nguyen QD, Brown DM, Marcus DM, et al. Ranibizumab for diabetic macular edema: results from 2 phase III randomized trials: RISE and RIDE. Ophthalmology. 2012;119(4):789-801.

77. Chen G, Tzekov R, Li W, Jiang F, Mao S, Tong Y. Subthreshold micropulse diode laser versus conventional laser photocoagulation for diabetic macular edema: a meta-analysis of randomized controlled trials. Retina. 2016;36(11):2059-65. 